\title{
Topological invariants in two-dimensional quasicrystals
}

\author{
Mikito Koshino (1) and Hiroki Oka (1) \\ Department of Physics, Osaka University, Osaka 560-0043, Japan
}

(Received 1 October 2021; accepted 21 December 2021; published 12 January 2022)

\begin{abstract}
We provide a topological concept to characterize energy gaps in general two-dimensional quasiperiodic systems. We show that every single gap is uniquely characterized by a set of integers, which quantize the area of the momentum space in units of multiple Brillouin zones. These integers are found to be equivalent to the second Chern numbers by considering a formal relationship between an adiabatic charge pumping under a potential sliding and the four-dimensional quantum Hall effect. The integers are independent of commensurability, and invariant under an arbitrary continuous deformation, such as a relative rotation of a twisted bilayer system.
\end{abstract}

DOI: 10.1103/PhysRevResearch.4.013028

\section{INTRODUCTION}

The topological phase of matter is a fundamental concept to understand the quantum properties in crystalline solids. The topological classification relies on the existence of a gap in the energy spectrum, where the topological invariant is determined by the dependence of eigenstates below the gap on the Bloch wave number $[1,2]$. The typical example is the integer quantum Hall effect, where the quantized Hall conductivity is expressed by the first Chern number, or an integral of the Berry curvature of the occupied states over the entire Brillouin zone $[3,4]$.

The topological properties of quasicrystalline systems have also attracted much attention. Quasiperiodic systems can also have energy gaps [5-8], while the lack of the Bloch bands makes the definition of topological numbers a nontrivial problem. Several theoretical works have been devoted to topological characterization of various one-dimensional (1D) [9-20] and two-dimensional (2D) quasiperiodic systems [21-28]. In particular, one-dimensional quasicrystals are characterized by adiabatic charge pumping, where the number of the transferred charge under a relative slide of a single periodic structure to the other is given by the first Chern number, in an analogous manner to the quantum Hall effect $[11,26,29,30]$.

Recent developments in the study of 2D materials gave rise to a new class of 2D quasicrystals controlled by twist [31-46]. When two atomic layers are overlaid with an arbitrary rotation angle, the periodicities of the individual layers do not generally match, and the entire system becomes quasiperiodic. A remarkable feature of these twisted 2D quasicrystals is that the electronic structure can be continuously modified by changing the twist angle or any deformations of the individual lattice structure. For instance, twisted bilayer graphene has a highly tunable band structure depending on the twist angle,

Published by the American Physical Society under the terms of the Creative Commons Attribution 4.0 International license. Further distribution of this work must maintain attribution to the author(s) and the published article's title, journal citation, and DOI. ranging from the moire flat bands in the small-angle regime [31-38,42-45], to a 12 -fold rotationally symmetric quasicrystal at $30^{\circ}[47-52]$.

Then, one may ask, what is a topological number to characterize energy gaps in a quasiperiodic system, which is invariant under a continuous structural deformation such as a relative rotation? In our previous work [53], we studied the energy spectrum in a quasiperiodic system of graphene sandwiched by hexagonal boron nitride (hBN) [54-61], and we found that each energy gap in the spectrum is uniquely characterized by a set of integers, which quantize the area of a quasi Brillouin zone in momentum space. These integers, which we refer to as zone quantum numbers, were shown to be invariant under interlayer rotation as long as the gap remains open. It implies that there should be an underlying topological mechanism that guarantees the quantization of the momentum space area, while the actual topological expression of the zone quantum numbers is yet to be cleared.

In this paper, we consider general 2D doubly periodic systems [i.e., a 2D Hamiltonian with periodic potentials $V^{\alpha}(\mathbf{r})$ and $V^{\beta}(\mathbf{r})$ which are generally incommensurate], and we show that six zone quantum numbers associated with every single energy gap turn out to be the second Chern numbers. The zone quantum numbers are simply defined as follows. The doubly periodic system has the redundant reciprocal-lattice vectors $\mathbf{b}_{1}, \ldots, \mathbf{b}_{4}\left(\mathbf{b}_{1}, \mathbf{b}_{2}\right.$ of $V^{\alpha}$, and $\mathbf{b}_{3}, \mathbf{b}_{4}$ of $\left.V^{\beta}\right)$ on the 2D plane, where we can define six fundamental Brillouin zones $S_{i j}^{*}=\left(\mathbf{b}_{i} \times \mathbf{b}_{j}\right)_{z}$ by taking the cross product of two distinct vectors [Fig. 1(a)]. Each energy gap is then characterized by six zone quantum numbers $v_{i j}$ such that the electron density below the gap is quantized as $n_{e}=\sum_{\langle i, j\rangle} v_{i j} S_{i j}^{*} /(2 \pi)^{2}$. The associated momentum area $\sum_{\langle i, j\rangle} v_{i j} S_{i j}^{*}$ corresponds to a quasi Brillouin zone, which is a single polygon composed of the composite Bragg planes given by $\mathbf{b}_{1}, \ldots, \mathbf{b}_{4}$, and it is a line of the gap opening momenta on the free-electron band in the infinitesimal potential limit [53].

We verify the equivalence between the zone quantum numbers $v_{i j}$ and the second Chern numbers by considering an adiabatic charge pumping under a relative slide of a periodic potential to the other (corresponding to interlayer sliding in twisted 2D materials). First, we demonstrate that the number 
(a)
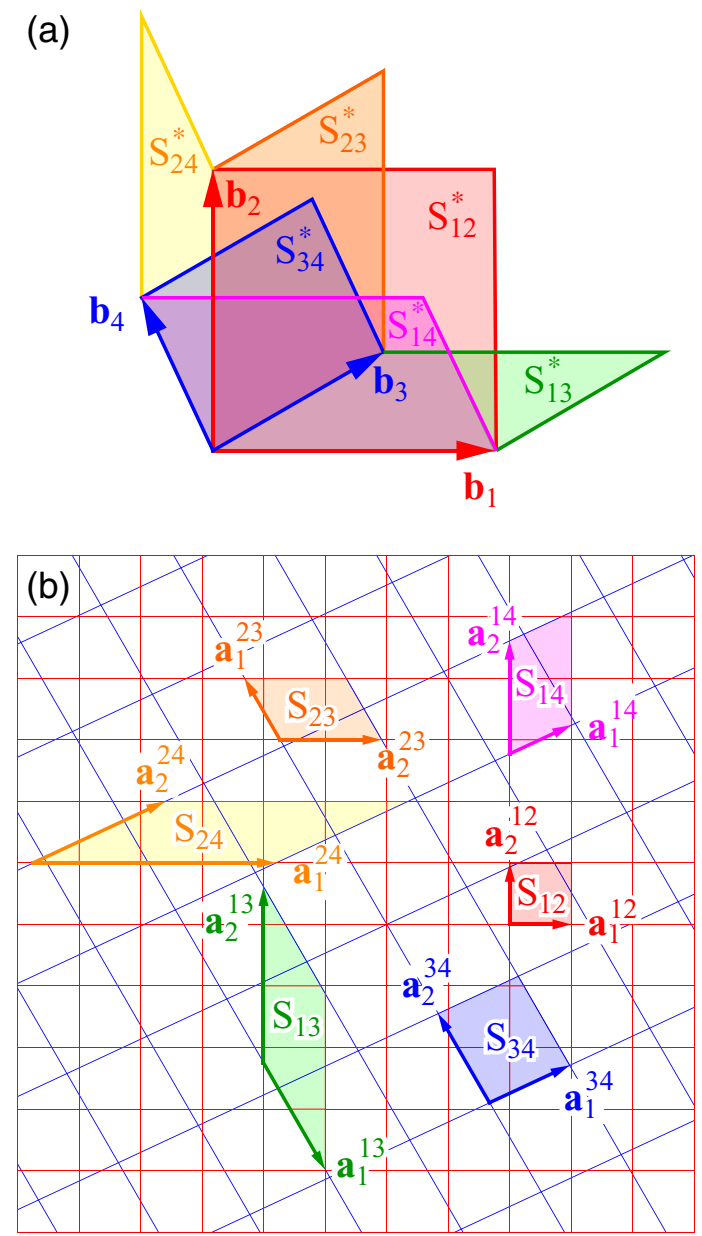

FIG. 1. (a) Momentum-space unit areas $S_{i j}^{*}=\left(\mathbf{b}_{i} \times \mathbf{b}_{j}\right)_{z}$. (b) Real-space unit areas $S_{i j}$ and the associated lattice vectors $\mathbf{a}_{1}^{i j}$ and $\mathbf{a}_{2}^{i j}$. The red (blue) grid lines represent the wave surfaces of $e^{i \mathbf{b}_{1} \cdot \mathbf{r}}$ and $e^{i \mathbf{b}_{2} \cdot \mathbf{r}}\left(e^{i \mathbf{b}_{3} \cdot \mathbf{r}}\right.$ and $\left.e^{i \mathbf{b}_{4} \cdot \mathbf{r}}\right)$.

of pumped charge is directly expressed in terms of the zone quantum numbers by a simple argument using the charge continuity in a potential deformation. Then we describe the same pumping process in an alternative approach using the dimensional reduction of the four-dimensional (4D) quantum Hall effect [21,62-67], where the charge pumping is expressed by the second Chern numbers. By comparing the corresponding equations in the two different approaches, we find that the zone quantum numbers are equivalent to the second Chern numbers. These integers are fixed in a continuous deformation of the potential, and they do not depend on the commensurability of the multiple periodicities.

The systematic characterization of energy gaps presented in this work would be applicable to general quasicrystalline systems having redundant reciprocal vectors more than the spatial dimension. The identification of the topological numbers in quasicrystalline systems bridges the fields of quasicrystal and topological condensed-matter physics.

The paper is organized as follows. In Sec. II, we present the formulation of the zone quantum numbers. We calculate the energy spectrum of a twisted double triangular potential as an example, and we identify the zone quantum numbers and the quasi Brillouin zones. In Sec. III, we consider the adiabatic charge pumping under a relative sliding of the double potential, and we show that the pumping charge is quantized by the zone quantum numbers. In Sec. IV, we present an alternative approach to describe the adiabatic charge pumping using the 4D quantum Hall effect, and we find the equivalence between the zone quantum numbers and the second Chern numbers. A brief conclusion is given in Sec. V.

\section{ZONE QUANTUM NUMBERS}

\section{A. General formulation}

We consider a doubly periodic 2D Hamiltonian

$$
H=\frac{\mathbf{p}^{2}}{2 m}+V^{\alpha}(\mathbf{r})+V^{\beta}(\mathbf{r}),
$$

where $V^{\lambda}(\mathbf{r})(\lambda=\alpha, \beta)$ is a periodic potential given by

$$
V^{\lambda}(\mathbf{r})=\sum_{m_{1}, m_{2}} V_{m_{1}, m_{2}}^{\lambda} e^{i\left(m_{1} \mathbf{b}_{1}^{\lambda}+m_{2} \mathbf{b}_{2}^{\lambda}\right) \cdot \mathbf{r}},
$$

and $\mathbf{b}_{1}^{\lambda}, \mathbf{b}_{2}^{\lambda}$ are its primitive reciprocal-lattice vectors. The real-space lattice vectors $\mathbf{a}_{1}^{\lambda}, \mathbf{a}_{2}^{\lambda}$ are defined such that $\mathbf{a}_{\mu}^{\lambda} \cdot \mathbf{b}_{v}^{\lambda}=$ $2 \pi \delta_{\mu \nu}$. It is useful to introduce serial indexes to label the four reciprocal-lattice vectors as

$$
\left(\mathbf{b}_{1}, \mathbf{b}_{2}, \mathbf{b}_{3}, \mathbf{b}_{4}\right)=\left(\mathbf{b}_{1}^{\alpha}, \mathbf{b}_{2}^{\alpha}, \mathbf{b}_{1}^{\beta}, \mathbf{b}_{2}^{\beta}\right) .
$$

We claim that, when the spectrum has an energy gap, the electron density below an energy gap is quantized as,

$$
n_{e}=\frac{1}{(2 \pi)^{2}} \sum_{\langle i, j\rangle} v_{i j} S_{i j}^{*}=\sum_{\langle i, j\rangle} \frac{v_{i j}}{S_{i j}} .
$$

Here $v_{i j}(i, j=1,2,3,4)$ are zone quantum numbers that characterize the gap, and $\langle i, j\rangle$ represents a pair of different indices. $S_{i j}^{*}$ and $S_{i j}$ are defined by

$$
S_{i j}^{*}=\left(\mathbf{b}_{i} \times \mathbf{b}_{j}\right)_{z}, \quad S_{i j}=(2 \pi)^{2} / S_{i j}^{*},
$$

where $(\cdots)_{z}$ represents the $z$-component perpendicular to the plane. $S_{i j}^{*}$ is a momentum space area spanned by two distinct reciprocal-lattice vectors chosen from $\mathbf{b}_{1}, \mathbf{b}_{2}, \mathbf{b}_{3}, \mathbf{b}_{4}$, and $S_{i j}$ is its real-space counterpart. We have six independent areas $S_{12}^{*}, S_{13}^{*}, S_{14}^{*}, S_{23}^{*}, S_{24}^{*}, S_{34}^{*}$ as illustrated in Fig. 1(a), and we have $S_{j i}^{*}=-S_{i j}^{*}$ and $S_{i i}^{*}=0$ from the definition. Accordingly, we have six zone quantum numbers $v_{12}, v_{13}, v_{14}, v_{23}, v_{24}, v_{34}$, and we define $v_{j i}=-v_{i j}$ and $v_{i i}=0$ for consistency. The areas $S_{i j}^{*}$ can be regarded as the projection of faces of a fourdimensional hypercube onto the physical 2D plane.

$S_{i j}$ is the area of the parallelogram formed by the wave surfaces of $e^{i \mathbf{b}_{i} \cdot \mathbf{r}}$ and $e^{i \mathbf{b}_{j} \cdot \mathbf{r}}$, as shown in Fig. 1(b). For later convenience, we define the lattice vectors

$$
\mathbf{a}_{1}^{i j}=\frac{S_{i j}}{2 \pi}\left(\mathbf{b}_{j} \times \mathbf{e}_{z}\right), \quad \mathbf{a}_{2}^{i j}=-\frac{S_{i j}}{2 \pi}\left(\mathbf{b}_{i} \times \mathbf{e}_{z}\right),
$$

where $\mathbf{e}_{z}$ is the unit vector perpendicular to the $2 \mathrm{D}$ plane. $\left(\mathbf{a}_{1}^{i j}, \mathbf{a}_{2}^{i j}\right)$ is the primitive lattice vector set corresponding to $\left(\mathbf{b}_{1}^{i j}, \mathbf{b}_{2}^{i j}\right) \equiv\left(\mathbf{b}_{i}, \mathbf{b}_{j}\right)$ in momentum space, and it spans the unit cell $S_{i j}=\left(\mathbf{a}_{1}^{i j} \times \mathbf{a}_{2}^{i j}\right)_{z}$, as illustrated in Fig. 1(b). The lattice vectors of the potential $\alpha$ and $\beta$ are given by $\mathbf{a}_{\mu}^{\alpha}=\mathbf{a}_{\mu}^{12}$ and $\mathbf{a}_{\mu}^{\beta}=\mathbf{a}_{\mu}^{34}$. 
(a) Single potential

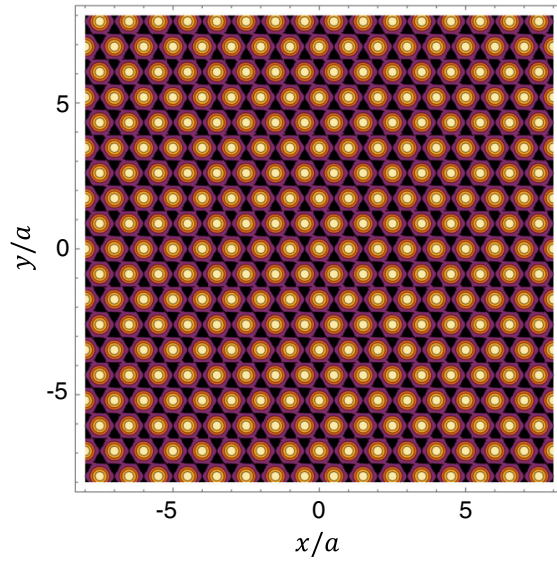

(b) $\theta=7^{\circ}$

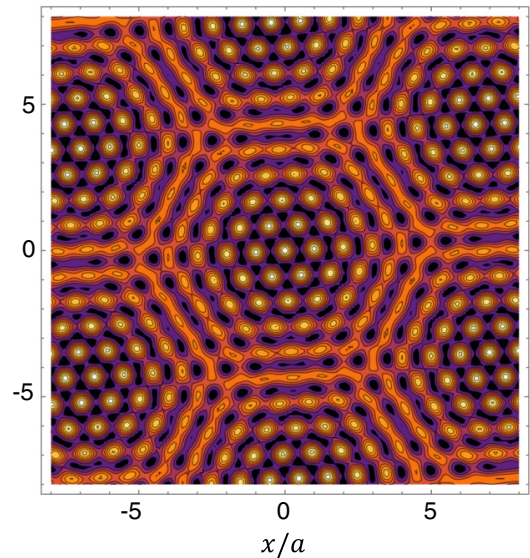

(c) $\theta=30^{\circ}$

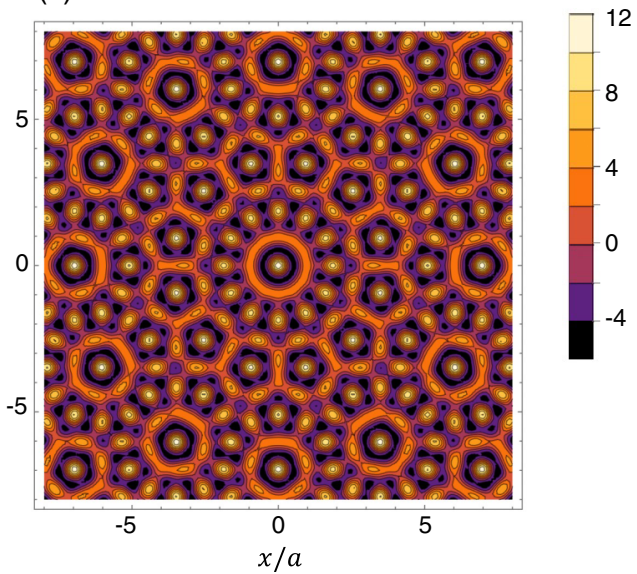

FIG. 2. Contour plots of (a) a single triangular potential, and (b) twisted double triangular potentials with $\theta=7^{\circ}$ and (c) $\theta=30^{\circ}$ [Eq. (7)].

\section{B. Example: Twisted triangular potentials}

In our previous work [53], we verified the relation Eq. (4) in a double-moiré system of graphene sandwiched by hexagonal boron nitride. In that system, only four areas out of the six $S_{i j}^{*}$ 's are independent due to the intrinsic $120^{\circ}$ rotational symmetry, and we identified the corresponding four zone quantum numbers for the energy gaps in the spectrum. The complete set of six integers can be obtained by allowing a slight deformation to break the symmetry. In the following, we demonstrate a full identification of the six characteristic integers in a double period system, Eq. (1), with a twisted double triangular potential. A similar calculation with a twisted square potential is presented in Appendix B.

The twisted double triangular potential is given by

$$
V^{\lambda}(\mathbf{r})=2 V_{0} \sum_{\mu=1}^{3} \cos \left[\mathbf{b}_{\mu}^{\lambda} \cdot\left(\mathbf{r}-\mathbf{r}_{0}^{\lambda}\right)\right],
$$

where $\mathbf{r}_{0}^{\lambda}$ is the origin of the potential $\lambda$. The reciprocal vectors of $\lambda=\alpha$ are given by

$$
\begin{aligned}
\mathbf{b}_{1}^{\alpha} & =\frac{2 \pi}{a}\left(\begin{array}{c}
1 \\
-1 / \sqrt{3}
\end{array}\right), \\
\mathbf{b}_{2}^{\alpha} & =\frac{2 \pi}{a}\left(\begin{array}{c}
0 \\
2 / \sqrt{3}
\end{array}\right), \\
\mathbf{b}_{3}^{\alpha} & =-\mathbf{b}_{1}^{\alpha}-\mathbf{b}_{2}^{\alpha},
\end{aligned}
$$

and those of $\beta$ are defined by

$$
\mathbf{b}_{\mu}^{\beta}=R(\theta) \mathbf{b}_{\mu}^{\alpha},
$$

where $R(\theta)$ is a $2 \mathrm{D}$ rotation matrix of angle $\theta$. The corresponding primitive lattice vectors are

$$
\begin{aligned}
& \mathbf{a}_{1}^{\alpha}=a\left(\begin{array}{l}
1 \\
0
\end{array}\right), \\
& \mathbf{a}_{2}^{\alpha}=a\left(\begin{array}{c}
1 / 2 \\
\sqrt{3} / 2
\end{array}\right), \\
& \mathbf{a}_{\mu}^{\beta}=R(\theta) \mathbf{a}_{\mu}^{\alpha} .
\end{aligned}
$$

The potential profile is presented in Fig. 2 for (a) a single potential, and (b) a double potential with $\theta=7^{\circ}$ and (c) $\theta=30^{\circ}$. In the following, the potential amplitude (identical in $\alpha$ and $\beta$ ) is taken as $V_{0}=0.213 \varepsilon_{0}$, where $\varepsilon_{0}=\hbar^{2} /\left(2 m a^{2}\right)$.

Generally, the potentials of $\alpha$ and $\beta$ do not have a common period, and hence the system does not have global translational symmetry. Here we calculate the energy spectrum by using commensurate approximants [53], which are obtained by slightly deforming the periodicity of $\alpha$ or $\beta$, so that the system has a finite super unit cell with an area $S_{\mathrm{c}}$. The details of the commensurate approximant method are described in Appendix A. We prepare a series of commensurate approximants to mimic the continuous rotation of the twist angle between the two potentials, and we calculate the energy bands and the density of states (DOS) for all the systems. For each approximant, we average the DOS over the relative translation $\Delta \mathbf{r}_{0}=\mathbf{r}_{0}^{\alpha}-\mathbf{r}_{0}^{\beta}$ to obtain a continuous spectrum as a function of the twist angle.

Figure 3(a) shows the density map of the DOS plotted against $\theta$ and energy, where the brighter color indicates larger DOS, and the dark blue region represents the gap. The array of bars in the upper part of the figure represents the commensurate approximants considered in the calculation. Figure 3(b) is the corresponding plot with the vertical axis converted to the electron density, where dots represent energy gaps with the gap width indicated by the size.

For each energy gap, the zone quantum numbers $v_{i j}$ can be identified in the following manner. In a commensurate approximant, the momentum space area $S_{i j}^{*}$ 's have the greatest common divisor $S_{\mathrm{c}}^{*}=(2 \pi)^{2} / S_{\mathrm{c}}$, and hence they are expressed as $S_{i j}^{*}=s_{i j} S_{\mathrm{c}}^{*}$ with integers $s_{i j}$. Also, the electron density below a given band gap is quantized as $n_{e}=\left[S_{\mathrm{c}}^{*} /(2 \pi)^{2}\right] r$, where $r$ is the number of occupied Bloch subbands. Then, Eq. (4) becomes the Diophantine equation $r=\sum_{\langle i, j\rangle} v_{i j} s_{i j}$. By considering more than six commensurate approximants sharing the same energy gap, the number of Diophantine equations can equal the number of approximants, and we finally obtain the integers $v_{i j}$ as a unique solution of the set of equations. It should also be noted that the original double triangular potential, Eq. (7), has constraints on $S_{i j}^{*}$ 's such as 


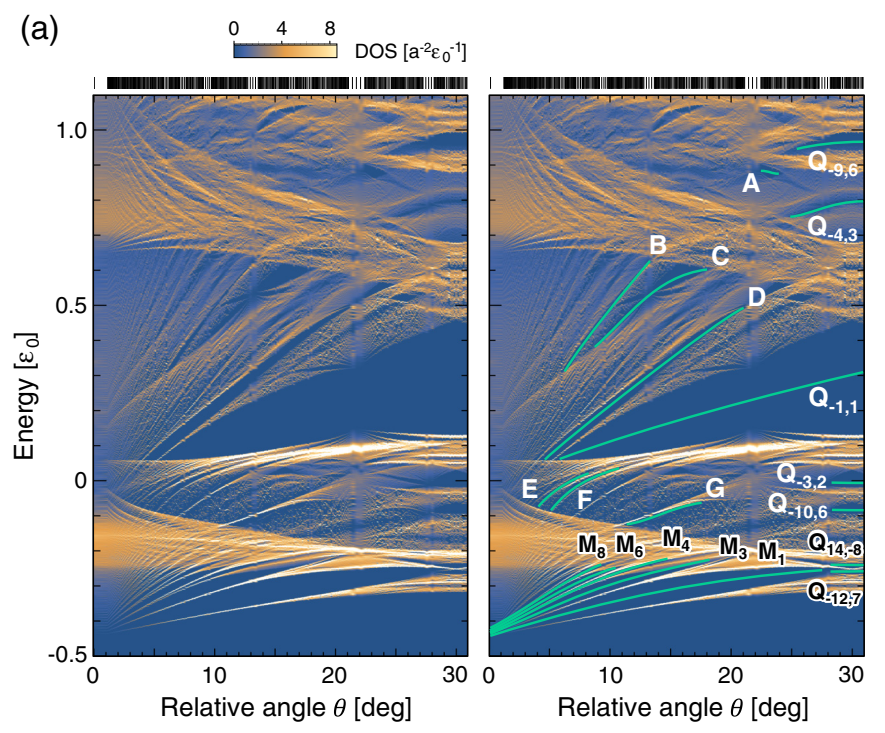

(b)

\begin{tabular}{|lllll|}
\hline$M_{1}:(1,0,-1,1,0,1)$ & $Q_{-9,6}:(-9,6,12,-6,6,-9)$ & $Q_{-10,6}:(-10,6,12,-6,6,-10)$ & $A:(-4,2,6,-4,2,-4)$ & $E:(14,-1,-14,13,-1,14)$ \\
$M_{3}:(3,0,-3,3,0,3)$ & $Q_{-4,3}:(-4,3,6,-3,3,-4)$ & $Q_{14,-8}:(14,-8,-16,8,-8,14)$ & $B:(-5,3,7,-4,3,-5)$ & $F:(11,-1,-11,10,-1,11)$ \\
$M_{4}:(4,0,-4,4,0,4)$ & $Q_{-1,1}:(-1,1,2,-1,1,-1)$ & $Q_{-12,7}:(-12,7,14,-7,7,-12)$ & $C:(-7,3,9,-6,3,-7)$ & $G:(4,-1,-4,3,-1,4)$ \\
$M_{6}:(6,0,-6,6,0,6)$ & $Q_{-3,2}:(-3,2,4,-2,2,-3)$ & & $D:(1,1,0,1,1,1)$ & \\
$M_{8}:(8,0,-8,8,0,8)$ & & & & \\
\hline
\end{tabular}

FIG. 3. (a) Density map of the DOS plotted against $\theta$ and energy in twisted double triangular potentials. The major gaps are highlighted and labeled in the right panel. The array of bars in the upper part of the figures represents the commensurate approximants considered in the calculation. (b) Corresponding plot with vertical axis converted to the electron density, where the dot size indicates the gap width. The bottom table presents the zone quantum numbers $\left(v_{12}, v_{13}, v_{14}, v_{23}, v_{24}, v_{34}\right)$ of the highlighted gaps.

$S_{12}^{*}=S_{34}^{*}$ and $S_{13}^{*}=S_{24}^{*}$ due to the high spatial symmetry, which prevents the full identification of $v_{i j}$ 's. This problem is removed by including systems with the symmetry slightly broken in the set of commensurate approximants. The actual calculation of the zone quantum numbers using the commensurate approximants is presented in Appendix A.

In the bottom of Fig. 3, we present the zone quantum numbers $\left(v_{12}, v_{13}, v_{14}, v_{23}, v_{24}, v_{34}\right)$ identified for some major gaps labeled in Figs. 3(a) and 3(b). The series $M_{n}$ in the low twist angle regime are the moiré gaps, which have the form

$$
M_{n}=n(1,0,-1,1,0,1) .
$$

In this region, the system is governed by a long-range moiré pattern as seen in Fig. 2(b), and the discrete levels separated by $M_{n}$ can be viewed as the Bloch subbands of the moiré superlattice. The reciprocal-lattice vectors for the moiré period are given by

$$
\mathbf{G}_{1}^{\mathrm{M}}=\mathbf{b}_{1}-\mathbf{b}_{3}, \quad \mathbf{G}_{2}^{\mathrm{M}}=\mathbf{b}_{2}-\mathbf{b}_{4},
$$

and the area of the moiré Brillouin zone becomes

$$
S_{\mathrm{M}}^{*}=\left(\mathbf{G}_{1}^{\mathrm{M}} \times \mathbf{G}_{2}^{\mathrm{M}}\right)_{z}=S_{12}^{*}-S_{14}^{*}+S_{23}^{*}+S_{34}^{*},
$$

which corresponds to $(1,0,-1,1,0,1)$. Equation (11) indicates that the momentum space area is quantized by $S_{\mathrm{M}}^{*}$.

In the large-angle region $\theta \gg 1^{\circ}$, the long-wavelength picture is no longer valid and the system cannot be effectively captured by any single periodicity. At $\theta=30^{\circ}$, in particular, the system becomes a quasicrystal with 12-fold rotational symmetry [47-52], as shown in Fig. 2(c). Here we find that the zone quantum numbers always have the form

$$
Q_{m, n}=(m, n, 2 n,-n, n, m) .
$$

The corresponding electronic density Eq. (4) is $n_{e}=(\sqrt{3} m+$ $3 n) / a^{2}$, indicating that there are two distinct units, $\sqrt{3} / a^{2}$ and $3 / a^{2}$, to quantize the electronic spectrum.

The constraint on the zone quantum numbers Eq. (14) is explained as follows. If we define $\mathbf{b}_{i}^{\prime}(i=1,2,3,4)$ by the $30^{\circ}$-rotation of $\mathbf{b}_{i}$, we have a relation $\left(\mathbf{b}_{1}^{\prime}, \mathbf{b}_{2}^{\prime}, \mathbf{b}_{3}^{\prime}, \mathbf{b}_{4}^{\prime}\right)=$ $\left(\mathbf{b}_{3}, \mathbf{b}_{4}, \mathbf{b}_{1}+\mathbf{b}_{2},-\mathbf{b}_{1}\right)$. The associated areas $S_{i j}^{* \prime}=\left(\mathbf{b}_{i}^{\prime} \times \mathbf{b}_{j}^{\prime}\right)_{z}$ can be expressed by the old areas as $S_{12}^{*}{ }^{\prime}=S_{34}^{*}, S_{13}^{*}{ }^{\prime}=-S_{13}^{*}-$ $S_{23}^{*}$, etc. When the system is invariant under the $30^{\circ}$ rotation, we should have $\sum_{\langle i, j\rangle} v_{i j} S_{i j}^{*}=\sum_{\langle i, j\rangle} v_{i j} S_{i j}^{* \prime}$ with the identical $v_{i j}$. By using the relationship between $S_{i j}^{* \prime}$ and $S_{i j}^{*}$, we obtain constraints for $\nu_{i j}$, and finally we find Eq. (14).

Other gaps are just labeled as $A, B, C, \ldots$ in Fig. 3. We see that the zone quantum numbers of any gaps definitely come in a form of $(m, n, r, n-r, n, m)$. This is explained by the coexistence of the $120^{\circ}$ rotational symmetry, which requires the form of ( $\left.m, n, r, n-r, n, m^{\prime}\right)$, and the reflection symmetry with respect to the in-plane axis between $\mathbf{b}_{1}$ and $\mathbf{b}_{3}$, which requires $\left(m, n, r, n-r, n^{\prime}, m\right)$. The constraints on the zone quantum numbers are proved by a similar argument to the 12-fold case.

In our previous work [53], we obtained only four zone quantum numbers in $\mathrm{hBN} /$ graphene/hBN systems because we only considered strictly $120^{\circ}$ symmetric commensurate approximants. The unit areas in those systems have the relationship $S_{24}^{*}=S_{13}^{*}$ and 
(a) $Q_{-1,1}:(-1,1,2,-1,1,-1)$
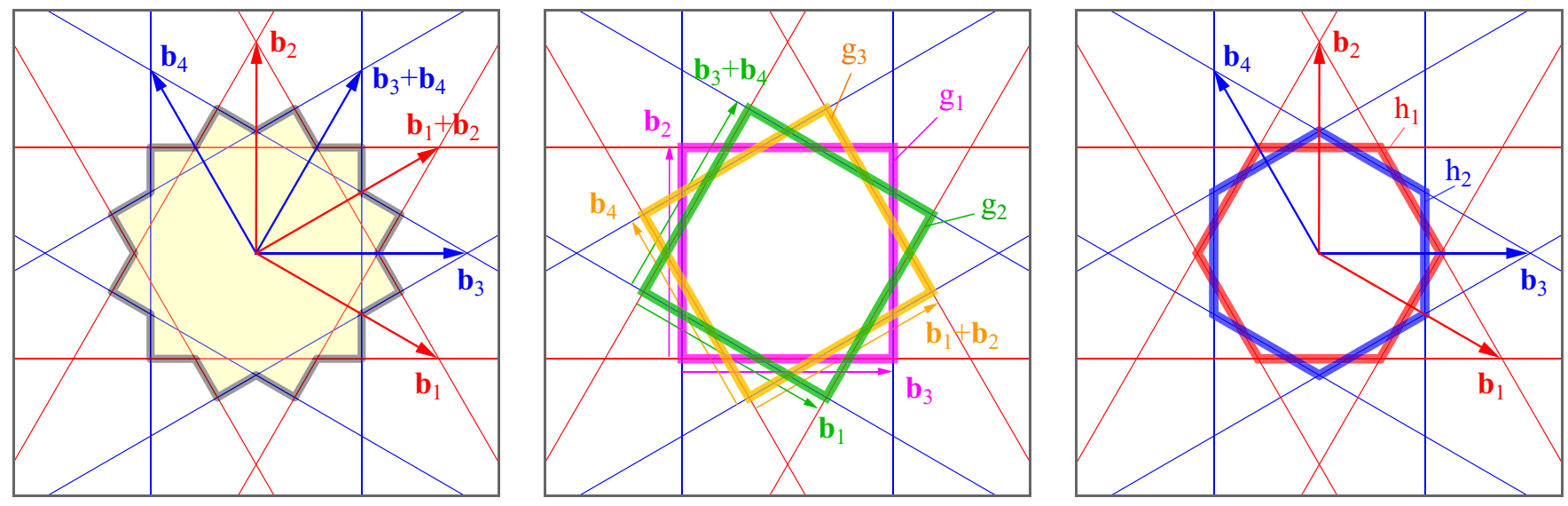

(b) $Q_{-3,2}:(-3,2,4,-2,2,-3)$
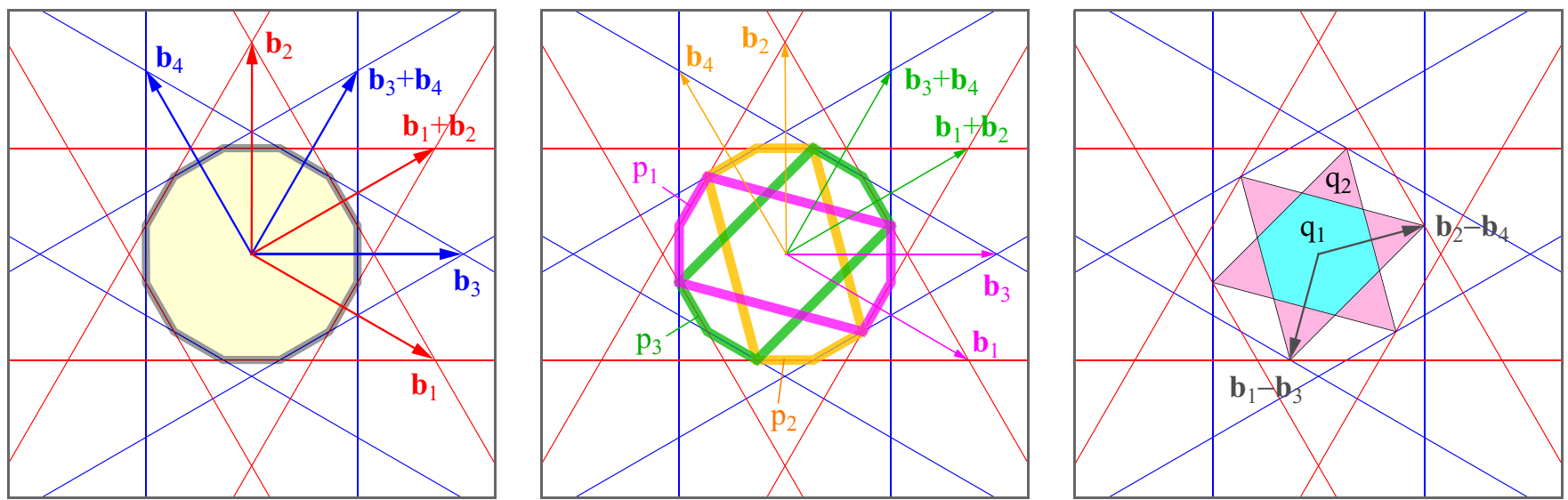

FIG. 4. The qBZs of (a) $Q_{-1,1}$ and (b) $Q_{-3,2}$ in the twisted triangular potential with $\theta=30^{\circ}$. The right two panels in each row illustrate the decomposition of the $\mathrm{qBZ}$ into the primitive Brillouin zones.

$S_{23}^{*}=-S_{13}^{*}-S_{14}^{*}$, so that $\sum_{\langle i, j\rangle} v_{i j} S_{i j}^{*}$ is reduced to $m_{1} S_{12}^{*}+m_{2} S_{34}^{*}+m_{3} S_{13}^{*}+m_{4} S_{14}^{*}$ with $\left(m_{1}, m_{2}, m_{3}, m_{4}\right)=$ $\left(v_{12}, v_{34}, v_{13}-v_{23}+v_{24}, v_{14}-v_{23}\right)$, which are the four integers defined in Ref. [53]. Recalling that $v_{i j}$ must have the form $\left(m, n, r, n-r, n, m^{\prime}\right)$ in $120^{\circ}$ symmetry, we can restore the complete six numbers $v_{i j}$ as $\left(m_{1}, \frac{2 m_{3}-m_{4}}{3}, \frac{m_{3}+m_{4}}{3}, \frac{m_{3}-2 m_{4}}{3}, \frac{2 m_{3}-m_{4}}{3}, m_{2}\right)$. The numbers are found to be integers for all the gaps identified in Ref. [53].

\section{Quasi Brillouin zones}

The area $\sum_{\langle i, j\rangle} v_{i j} S_{i j}^{*}$ can be associated with a geometric object in momentum space referred to as the quasi Brillouin zone (qBZ). The boundary qBZ for a given gap is defined as a set of $k$-points on the original free-electron band, at which the gap starts to open in the infinitesimal potential limit [53]. Generally, the qBZ is a polygon composed of multiple segments of Bragg planes, which are the perpendicular bisectors of composite reciprocal-lattice vectors $\mathbf{G}=m_{1} \mathbf{b}_{1}+m_{2} \mathbf{b}_{2}+$ $m_{3} \mathbf{b}_{3}+m_{4} \mathbf{b}_{4}$.

Let us consider the twisted triangular potential considered in the previous section. The qBZ for the moiré gap $M_{n}$ is found to be just the $n$th Brillouin zone defined by the moiré reciprocal vectors $\mathbf{G}_{1}^{\mathrm{M}}$ and $\mathbf{G}_{2}^{\mathrm{M}}$ [Eq. (12)]. In general twist angles, however, the qBZ does not coincide with any Brillouin zone of a periodic system. We show the qBZs of the gap $Q_{-1,1}$ and $Q_{-3,2}$ at $\theta=30^{\circ}$ in the leftmost panels of Figs. 4(a) and 4(b), respectively. The areas of these qBZs can be easily calculated by the decomposition illustrated in the right two panels. For instance, the area of the qBZ for gap $Q_{-1,1}$ [Fig. 4(a)] is expressed by three squares, $g_{1}, g_{2}, g_{3}$, and two hexagons, $h_{1}, h_{2}$, as $S^{*}\left(Q_{-1,1}\right)=\left(g_{1}+g_{2}+g_{3}\right)-\left(h_{1}+\right.$ $\left.h_{2}\right)$. The area $g_{1}$ is given by $g_{1}=\left(\mathbf{b}_{3} \times \mathbf{b}_{2}\right)_{z}=-S_{23}^{*}$, and similarly we have $g_{2}=S_{14}^{*}+S_{24}^{*}, g_{3}=S_{13}^{*}+S_{14}^{*}, h_{1}=S_{12}^{*}$, and $h_{2}=S_{34}^{*}$. Finally, we have $S^{*}\left(Q_{-1,1}\right)=-S_{12}^{*}+S_{13}^{*}+$ $2 S_{14}^{*}-S_{23}^{*}+S_{24}^{*}-S_{34}^{*}$, which agrees with the zone quantum numbers $(-1,1,2,-1,1,-1)$ obtained in the previous section.

Similarly, the area of the qBZ for gap $Q_{-3,2}$ is expressed by $S^{*}\left(Q_{-3,2}\right)=p_{1}+p_{2}+p_{3}-2 q_{1}-q_{2}$ as shown Fig. 4(b). The area $p_{1}$ is the Wigner-Seitz cell in the reciprocal lattice of $\mathbf{b}_{1}$ and $\mathbf{b}_{3}$, and hence $p_{1}=S_{13}^{*}$. The $q_{1}$ (hexagon) and $q_{2}$ (six triangles) are the first and second Brillouin zones defined by the primitive vectors $\mathbf{b}_{1}-\mathbf{b}_{3}$ and $\mathbf{b}_{2}-\mathbf{b}_{4}$, and therefore $q_{1}=$ $q_{2}=\left[\left(\mathbf{b}_{1}-\mathbf{b}_{3}\right) \times\left(\mathbf{b}_{2}-\mathbf{b}_{4}\right)\right]_{z}=S_{12}^{*}+S_{34}^{*}-S_{14}^{*}+S_{23}^{*}$. As a result, the area $S^{*}\left(Q_{-3,2}\right)$ becomes $(-3,2,4,-2,2,-3)$.

At $30^{\circ}$, we have symmetry constraints such as $g_{1}=g_{2}=$ $g_{3}$, and one might think the decomposition of the $\mathrm{qBZ}$ area 


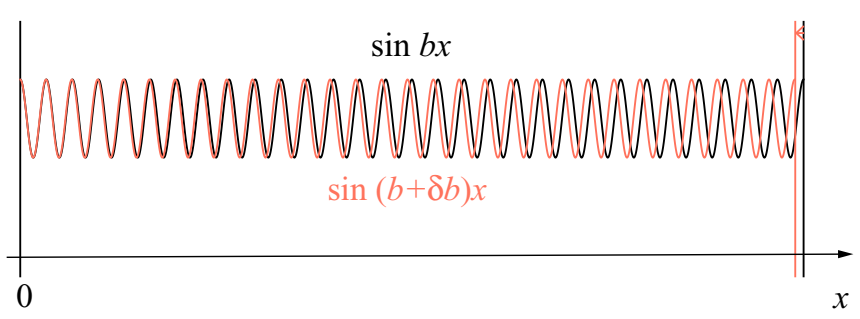

FIG. 5. Schematic picture of an adiabatic process slightly changing the wave number of a 1D periodic potential. At a point far from the origin $(|x| \gg 2 \pi / b)$, the change can be viewed as a parallel translation of the potential.

into $S_{i j}^{*}$ 's is not unique. However, the area quantization with the same $v_{i j}$ strictly holds when the potential is deformed to break the symmetry, and this guarantees a uniqueness of the decomposition.

\section{ADIABATIC CHARGE PUMPING}

Here we show that the zone quantum numbers introduced in the previous section characterize the adiabatic charge pumping under the relative sliding of the doubly periodic potential.

\section{A. 1D systems}

We first consider a doubly periodic 1D Hamiltonian

$$
H=\frac{p^{2}}{2 m}+V_{1}(x)+V_{2}(x)
$$

where $V_{i}(x)=\sum_{m} V_{i, m} e^{i m b_{i} x}(i=1,2)$ is a periodic potential with the period of $a_{i}=2 \pi / b_{i}$. Now we consider a cyclic process where one of the periodic potential $V_{i}(x)$ is adiabatically translated by its period $a_{i}$, with the other fixed. The translated potential is expressed as

$$
V_{i}\left(x-\frac{\phi_{i}}{2 \pi} a_{i}\right)=\sum_{m} V_{i, m} e^{i m\left(b_{i} x-\phi_{i}\right)},
$$

where an increase of $\phi_{i}$ from 0 to $2 \pi$ gives a unit slide of $V_{i}(x)$ by distance $a_{i}$.

We define $\Delta P_{i}$ by the change of the electric polarization during a unit slide. In $1 \mathrm{D}$, the $\Delta P_{i}$ has a dimension of the electronic density (number of electrons per unit length) times length, which is dimensionless. Note that we exclude the electric charge $-e$ in the definition of the polarization. Here we claim the following: When the Fermi energy is in a gap, the polarization change per cycle, $\Delta P_{i}$, and the electron density below the gap, $n_{e}$, are related by

$$
\Delta P_{i}=2 \pi \frac{\partial n_{e}}{\partial b_{i}} .
$$

Equation (17) can be proved by the following consideration. Let us consider an adiabatic process where the wave number $b_{i}$ is slightly changed to $b_{i}+\delta b_{i}$. As illustrated in Fig. 5, the corresponding change of $V_{i}(x)$ at a point far from the origin $\left(|x| \gg a_{i}\right)$ can be viewed as a parallel translation of the fixed potential $V_{i}(x)$. Considering the phase factor $b_{i} x-\phi_{i}$ in Eq. (16), the change of $b_{i}$ to $b_{i}+\delta b_{i}$ can be absorbed to the change of $\phi_{i}$ by $\delta \phi_{i}=-\delta b_{i} x$, and this gives the phase shift in the effective translation at $x$. Since this process amounts to $n=\delta \phi_{i} /(2 \pi)$ cycles of a unit translation, the number of electrons passing through the point $x$ is given by $n \Delta P_{i}=$ $-\Delta P_{i} \delta b_{i} x /(2 \pi)$. Due to the continuity of the electric charge, it must be equal to the change of the number of electrons in a region from 0 to $x$. This leads to a relation $-\Delta P_{i} \delta b_{i} x /(2 \pi)=$ $-x \delta n_{e}$, and we obtain Eq. (17).

In the doubly periodic system given by Eq. (15), every gap in the spectrum is characterized by a pair of integers $m_{1}$ and $m_{2}$, such that the electron density below the gap is given by

$$
n_{e}=\frac{1}{2 \pi}\left(m_{1} b_{1}+m_{2} b_{2}\right)=\frac{m_{1}}{a_{1}}+\frac{m_{2}}{a_{2}} .
$$

By using Eq. (17), we conclude $\Delta P_{i}=m_{i}$, i.e., $m_{i}$ electrons passed through any cross section of the system. The integers $m_{1}$ and $m_{2}$ coincide with the first Chern numbers $[11,26,29,30]$, as presented in Appendix A.

\section{B. 2D systems}

The same argument is available for a doubly periodic $2 \mathrm{D}$ Hamiltonian, Eq. (1). We consider an adiabatic translation of the periodic potential $V^{\lambda}(\lambda=\alpha$ or $\beta)$ by $\mathbf{a}_{\mu}^{\lambda}$, and we calculate the change of the electric polarization during the process. A parallel translation of $V^{\lambda}(\mathbf{r})$ is expressed as

$$
\begin{aligned}
V^{\lambda} & \left(\mathbf{r}-\frac{\phi_{1}^{\lambda}}{2 \pi} \mathbf{a}_{1}^{\lambda}-\frac{\phi_{2}^{\lambda}}{2 \pi} \mathbf{a}_{2}^{\lambda}\right) \\
& =\sum_{m_{1}, m_{2}} V_{m_{1}, m_{2}}^{\lambda} e^{i m_{1}\left(\mathbf{b}_{1}^{\lambda} \cdot \mathbf{r}-\phi_{1}^{\lambda}\right)+i m_{2}\left(\mathbf{b}_{2}^{\lambda} \cdot \mathbf{r}-\phi_{2}^{\lambda}\right)},
\end{aligned}
$$

where we used $\mathbf{a}_{\mu}^{\lambda} \cdot \mathbf{b}_{v}^{\lambda}=2 \pi \delta_{\mu \nu}$. An increase of $\phi_{\mu}^{\lambda}$ from 0 to $2 \pi$ gives a unit translation of the potential $V^{\lambda}$ by $\mathbf{a}_{\mu}^{\lambda}$.

The situation can be systematically described by a generalized Hamiltonian $H=\mathbf{p}^{2} /(2 m)+V$ with

$$
V\left(\mathbf{r} ; \phi_{1}, \ldots, \phi_{N}\right)=\sum_{m_{1}, \ldots, m_{N}} V_{m_{1}, \ldots, m_{N}} e^{i \sum_{i=1}^{N} m_{i}\left(\mathbf{b}_{i} \cdot \mathbf{r}-\phi_{i}\right)} .
$$

The current double-period system corresponds to $N=4$, where $\mathbf{b}_{1}, \ldots, \mathbf{b}_{4}$ are given by Eq. (3), and

$$
\left(\phi_{1}, \phi_{2}, \phi_{3}, \phi_{4}\right)=\left(\phi_{1}^{\alpha}, \phi_{2}^{\alpha}, \phi_{1}^{\beta}, \phi_{2}^{\beta}\right) .
$$

We consider a cyclic process where $\phi_{i}$ of a certain $i$ is adiabatically increased from 0 to $2 \pi$. When the Fermi energy is in a gap, we can show that the change of the electric polarization during the process is

$$
\Delta \mathbf{P}_{i}=2 \pi \frac{\partial n_{e}}{\partial \mathbf{b}_{i}}
$$

which is the $2 \mathrm{D}$ version of Eq. (17). Now $\Delta \mathbf{P}_{i}$ has a dimension of the electronic density (number of electrons per unit area) times length.

Equation (22) is derived as follows. Let us consider the change of the potential $V(\mathbf{r})$ when $\mathbf{b}_{i}$ is changed to $\mathbf{b}_{i}+\delta \mathbf{b}_{i}$. In a similar manner to the $1 \mathrm{D}$ case, the change at a point far from the origin $\left(|\mathbf{r}| \gg 2 \pi /\left|\mathbf{b}_{i}\right|\right)$ is equivalent to a parallel translation of $\delta \phi_{i}=-\delta \mathbf{b}_{i} \cdot \mathbf{r}$, noting the phase factor $\mathbf{b}_{i} \cdot \mathbf{r}-\phi_{i}$ in Eq. (20). This causes a polarization change by $\Delta \mathbf{P}_{i} \delta \phi_{i} /(2 \pi)=\Delta \mathbf{P}_{i}\left(-\delta \mathbf{b}_{i} \cdot \mathbf{r}\right) /(2 \pi)$ at the point $\mathbf{r}$. The number of electrons passing through a line segment from $\mathbf{r}$ to 
$\mathbf{r}+d \mathbf{r}$ is given by

$$
d N_{e}=\left[\left(d \mathbf{r} \times \mathbf{e}_{z}\right) \cdot \Delta \mathbf{P}_{i}\right]\left(\delta \mathbf{b}_{i} \cdot \mathbf{r}\right) /(2 \pi) .
$$

Now we consider a large closed path $C$ on the 2D plane, and let $N_{e}$ be the number of electrons inside $C$. When $\mathbf{b}_{i}$ is changed to $\mathbf{b}_{i}+\delta \mathbf{b}_{i}$, the change of $N_{e}$ is calculated by integrating Eq. (23) along the path to obtain

$$
\begin{aligned}
\delta N_{e} & =\oint_{C} d N_{e}=\frac{1}{2 \pi} \oint_{C}\left[\left(d \mathbf{r} \times \mathbf{e}_{z}\right) \cdot \Delta \mathbf{P}_{i}\right]\left(\delta \mathbf{b}_{i} \cdot \mathbf{r}\right) \\
& =\frac{S}{2 \pi} \Delta \mathbf{P}_{i} \cdot \delta \mathbf{b}_{i},
\end{aligned}
$$

where $S$ is the area of the region enclosed by $C$, and we used the relationship $\oint_{C}\left(d \mathbf{r} \times \mathbf{e}_{z}\right)_{\mu} r_{v}=S \delta_{\mu \nu}$ in 2D. Since $n_{e}=$ $N_{e} / S$, we end up with Eq. (22). Alternatively, Eq. (22) can also be derived in the infinitesimal potential limit by integrating the Berry curvature on the boundary of the quasi Brillouin zone. A detailed argument is presented in Appendix C.

In a 2D doubly periodic system, the electron density below an energy gap is quantized by Eq. (4), as argued in the previous section. By applying the formula Eq. (22) to Eq. (4), the charge pumping $\Delta \mathbf{P}_{i}$ is explicitly calculated as

$$
\begin{aligned}
\Delta \mathbf{P}_{i} & =\frac{1}{2 \pi} \sum_{\langle k, j\rangle} v_{k j} \frac{\partial S_{k j}^{*}}{\partial \mathbf{b}_{i}} \\
& =\frac{1}{2 \pi} \sum_{j} v_{i j}\left(\mathbf{b}_{j} \times \mathbf{e}_{z}\right),
\end{aligned}
$$

where we used $S_{i j}^{*}=\left(\mathbf{b}_{i} \times \mathbf{b}_{j}\right) \cdot \mathbf{e}_{z}=\left(\mathbf{b}_{j} \times \mathbf{e}_{z}\right) \cdot \mathbf{b}_{i}$.

By using the real-space lattice vectors Eq. (6), Eq. (25) can also be written as

$$
\Delta \mathbf{P}_{i}=\sum_{j} \frac{v_{i j}}{S_{i j}} \mathbf{a}_{1}^{i j}
$$

The physical interpretation of Eq. (26) is as follows. Equation (4) states that $v_{i j}$ electrons reside in each unit area of $S_{i j}$. When $\phi_{1}$ is changed from 0 to $2 \pi$ (i.e., $V^{\alpha}$ is slid by $\mathbf{a}_{1}^{\alpha}$ ), for instance, the wave surface of $\mathbf{b}_{1}$ is moved by its single period, resulting in shifts of the unit areas $S_{12}, S_{13}, S_{14}$ by $\mathbf{a}_{1}^{12}, \mathbf{a}_{1}^{13}, \mathbf{a}_{1}^{14}$, respectively [see Fig. 1(b)]. For each of $j=2,3,4$, the electron density of $v_{1 j} / S_{1 j}$ is transferred by $\mathbf{a}_{1}^{1 j}$, resulting in a polarization change by $\Delta \mathbf{P}_{1}=$ $\sum_{j=2,3,4}\left(v_{1 j} / S_{1 j}\right) \mathbf{a}_{1 j}^{1 j}$.

\section{Example: Twisted triangular potentials}

As an example, we consider the adiabatic pumping in the twisted triangular potential in Sec. II B. For the moiré gap $M_{n}=n(1,0,-1,1,0,1)$ [Eq. (11)], Eq. (25) immediately leads to the equations

$$
\begin{aligned}
& \Delta \mathbf{P}_{1}=\frac{n}{2 \pi}\left(\mathbf{b}_{2}-\mathbf{b}_{4}\right) \times \mathbf{e}_{z}=\frac{n}{S_{\mathrm{M}}} \mathbf{L}_{1}^{\mathrm{M}}, \\
& \Delta \mathbf{P}_{2}=\frac{n}{2 \pi}\left(\mathbf{b}_{1}-\mathbf{b}_{3}\right) \times \mathbf{e}_{z}=\frac{n}{S_{\mathrm{M}}} \mathbf{L}_{2}^{\mathrm{M}}, \\
& \Delta \mathbf{P}_{3}=-\frac{n}{2 \pi}\left(\mathbf{b}_{2}-\mathbf{b}_{4}\right) \times \mathbf{e}_{z}=-\frac{n}{S_{\mathrm{M}}} \mathbf{L}_{1}^{\mathrm{M}}, \\
& \Delta \mathbf{P}_{4}=-\frac{n}{2 \pi}\left(\mathbf{b}_{1}-\mathbf{b}_{3}\right) \times \mathbf{e}_{z}=-\frac{n}{S_{\mathrm{M}}} \mathbf{L}_{2}^{\mathrm{M}},
\end{aligned}
$$

where $\mathbf{L}_{i}^{\mathrm{M}}$ is the moiré lattice vector defined by

$$
\mathbf{L}_{1}^{\mathrm{M}}=\frac{S_{\mathrm{M}}}{2 \pi}\left(\mathbf{G}_{2}^{\mathrm{M}} \times \mathbf{e}_{z}\right), \quad \mathbf{L}_{2}^{\mathrm{M}}=-\frac{S_{\mathrm{M}}}{2 \pi}\left(\mathbf{G}_{1}^{\mathrm{M}} \times \mathbf{e}_{z}\right),
$$

and we used Eq. (12). This indicates that, when the potential $\alpha(\beta)$ is slid by its unit vector $\mathbf{a}_{\mu}^{\alpha}\left(\mathbf{a}_{\mu}^{\beta}\right)$, then $n$ electrons per the moiré unit cell are pumped by a moiré unit vector $n \mathbf{L}_{\mu}^{\mathrm{M}}\left(-n \mathbf{L}_{\mu}^{\mathrm{M}}\right)$. The result coincides with the adiabatic moiré pumping in the previous works [26-28].

The argument is also applicable to the quasicrystal gaps at $\theta=30^{\circ}$. Here the zone quantum numbers take the form $Q_{m, n}=(m, n, 2 n,-n, n, m)$ [Eq. (14)]. For $\Delta \mathbf{P}_{1}$, for instance, Eq. (25) gives

$$
\begin{aligned}
\Delta \mathbf{P}_{1} & =\frac{1}{2 \pi}\left(m \mathbf{b}_{2}+n \mathbf{b}_{3}+2 n \mathbf{b}_{4}\right) \times \mathbf{e}_{z} \\
& =\frac{1}{L}(m+\sqrt{3} n)\left(\begin{array}{l}
1 \\
0
\end{array}\right)=\frac{n_{e}}{2} \mathbf{a}_{1}^{\alpha} .
\end{aligned}
$$

In the last equation, we used Eq. (10) and note that the electronic density [Eq. (4)] is $n_{e}=(\sqrt{3} m+3 n) / L^{2}$ at $\theta=30^{\circ}$. By similar calculations, we have a set of equations independent of $m$ and $n$,

$$
\begin{array}{rlrl}
\Delta \mathbf{P}_{1} & =\frac{n_{e}}{2} \mathbf{a}_{1}^{\alpha}, & \Delta \mathbf{P}_{2}=\frac{n_{e}}{2} \mathbf{a}_{2}^{\alpha}, \\
\Delta \mathbf{P}_{3}=\frac{n_{e}}{2} \mathbf{a}_{1}^{\beta}, & \Delta \mathbf{P}_{4}=\frac{n_{e}}{2} \mathbf{a}_{2}^{\beta} .
\end{array}
$$

From the definition, $\Delta \mathbf{P}_{1}$ and $\Delta \mathbf{P}_{2}\left(\Delta \mathbf{P}_{3}\right.$ and $\left.\Delta \mathbf{P}_{4}\right)$ represent the polarization changes when the potential $\alpha(\beta)$ is translated by $\mathbf{a}_{1}^{\alpha}$ and $\mathbf{a}_{2}^{\alpha}\left(\mathbf{a}_{1}^{\beta}\right.$ and $\left.\mathbf{a}_{2}^{\beta}\right)$, respectively. Equation (30) shows that, in any sliding processes, the transfer of the electric charge is always parallel to the potential sliding direction (regardless of which potential we move), and that the amount of the charge pumping is equivalent to the movement of half of the total electric charge by the sliding vector.

\section{4D QUANTUM HALL EFFECT AND THE SECOND CHERN NUMBERS}

In the following, we describe the adiabatic pumping argued in the previous section in an alternative approach using the dimensional reduction of the four-dimensional (4D) quantum Hall effect (QHE), [21,62-67], and we demonstrate that the zone quantum number $v_{i j}$ coincides with the second Chern number.

We first consider the 3D QHE as a simple example. Let us consider an infinite stack of 2D free-electron systems as illustrated Fig. 6(a), which is continuous in the $x$ and $y$ directions and discrete in the $z$ direction with lattice spacing $a_{z}$. For the $z$ direction, we assume nearest-neighbor tight-binding coupling $t_{z}$ between adjacent layers. We apply a magnetic field $B_{\mu \nu}=\partial_{\mu} A_{\nu}-\partial_{\nu} A_{\mu}$. Here we assume a uniform, inplane field $\left(B_{y z}, B_{z x}, 0\right)$, and we set the vector potential as $\mathbf{A}=\left(0,0, A_{z}\right)$ with $A_{z}=B_{x z} x+B_{y z} y$ (note $\left.B_{x z}=-B_{z x}\right)$. The motion of an electron is described by the Schrödinger equation,

$$
\begin{aligned}
& \frac{\mathbf{p}^{2}}{2 m} \Psi(x, y, z)-t_{z}\left[e^{i \frac{e}{\hbar} A_{z} a_{z}} \Psi\left(x, y, z+a_{z}\right)\right. \\
& \left.\quad+e^{-i \frac{e}{\hbar} A_{z} a_{z}} \Psi\left(x, y, z-a_{z}\right)\right]=E \Psi(x, y, z),
\end{aligned}
$$


(a)
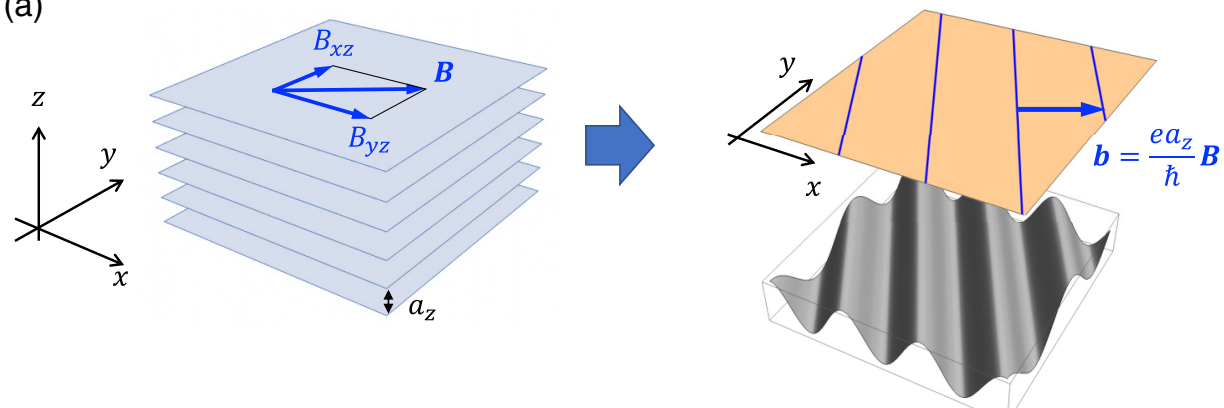

(b)

$$
\boldsymbol{B}_{i}=\left(B_{x i}, B_{y i}\right)(i=1,2,3,4)
$$
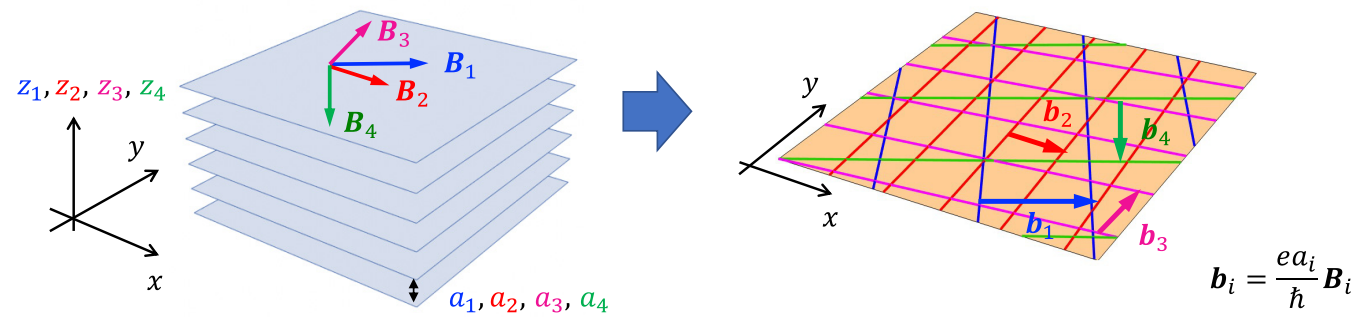

FIG. 6. (a) Left: three-dimensional stack of 2D free-electron systems under in-plane magnetic field. Note that the vector $\mathbf{B}$ is given by $\mathbf{B}=\left(B_{y z}, B_{x z}\right)=\left(B_{x},-B_{y}\right)$, which is not the natural expression of the magnetic field $\left(B_{x}, B_{y}\right)$. Right: the corresponding $2 \mathrm{D}$ system after the dimensional reduction (see the text). The wave vector $\mathbf{b}$ of the sinusoidal wave is proportional to $\mathbf{B}$ in the left panel. (b) Six-dimensional stack of 2D free-electron systems under in-plane magnetic field (left) and the corresponding 2D system with four sinusoidal potentials (right).

where $\mathbf{p}=-i \hbar\left(\partial_{x}, \partial_{y}\right)$ is the in-plane momentum. Since the Hamiltonian is periodic in $z$, the wave function can be factorized as $\Psi(x, y, z)=\psi(x, y) e^{i k_{z} z}$, and then Eq. (31) is reduced to a $2 \mathrm{D}$ Schrödinger equation,

$$
\frac{\mathbf{p}^{2}}{2 m} \psi-2 t_{z} \cos \left(\mathbf{b} \cdot \mathbf{x}+\phi_{z}\right) \psi=E \psi
$$

where $\mathbf{b}=\left(e a_{z} / \hbar\right)\left(B_{x z}, B_{y z}\right), \mathbf{x}=(x, y)$, and $\phi_{z}=k_{z} a_{z}$. This is a $2 \mathrm{D}$ system with a single sinusoidal potential with the wave number $\mathbf{b}$. The phase factor $\phi_{z}$ corresponds to the wave number in the $z$ direction.

The extension of higher dimensions is straightforward. We consider a six-dimensional (6D) system in $\left(x, y, z_{1}, z_{2}, z_{3}, z_{4}\right)$ space, which is continuous in the $x$ and $y$ directions and discrete in the $z_{i}(i=1,2,3,4)$ direction. We apply a uniform magnetic field $B_{y i}$ and $B_{i x}\left(=-B_{x i}\right)$ on the $y z_{i}$-plane and the $z_{i} x$-plane, respectively, and we take the vector potential $\mathbf{A}=\sum_{i=1}^{4}\left(B_{x i} x+B_{y i} y\right) \mathbf{e}_{i}$, where $\mathbf{e}_{i}$ is the unit vector in the $z_{i}$ direction. The system is schematically illustrated Fig. 6(b). As the Hamiltonian is periodic in any $z_{i}$ 's, the wave function can be written as $\Psi\left(x, y, z_{1}, z_{2}, z_{3}, z_{4}\right)=\psi(x, y) e^{i \sum_{i} k_{i} z_{i}}$, where $k_{i}$ is the Bloch wave number defined in $\pi / a_{i}<k_{i} \leqslant \pi / a_{i}$. The $6 \mathrm{D}$ Schrödinger equation is reduced to $(x, y)$ space as

$$
\frac{\mathbf{p}^{2}}{2 m} \psi-\sum_{i=1}^{4} 2 t_{i} \cos \left(\mathbf{b}_{i} \cdot \mathbf{x}+\phi_{i}\right) \psi=E \psi,
$$

where

$$
\mathbf{b}_{i}=\frac{e a_{i}}{\hbar}\left(B_{x i}, B_{y i}\right), \quad \phi_{i}=k_{i} a_{i} .
$$

This is equivalent to the double-period 2D system considered in this paper. The higher harmonic terms in $\mathbf{b}_{i}$ can be incorporated by assuming further layer hopping in the $z_{i}$ direction.
The electromagnetic response of the system is characterized by the second Chern number [21,62-67]. Let us consider a commensurate approximant where the periodicities of $\mathbf{b}_{i}(i=1,2,3,4)$ have a common super unit cell, and define the Bloch wave number $\left(k_{x}, k_{y}\right)$ in the corresponding super Brillouin zone. The Bloch Hamiltonian for the $6 \mathrm{D}$ system is written as $H\left(k_{x}, k_{y}, k_{1}, k_{2}, k_{3}, k_{4}\right)$. We consider the 4D subspace $k_{\mu}=\left(k_{x}, k_{y}, k_{i}, k_{j}\right)$ by choosing two indexes $i, j$ from 1 to 4 , with the remaining two wave numbers fixed. When the spectrum of the 4D Hamiltonian $H\left(k_{x}, k_{y}, k_{i}, k_{j}\right)$ is gapped, the second Chern number for the gap is defined as [21,6267],

$$
C_{i j}^{(2)}=\frac{1}{32 \pi^{2}} \int_{\mathrm{BZ}} d^{4} k \epsilon^{\mu \nu \lambda \rho} \operatorname{Tr}\left[\mathcal{F}_{\mu \nu} \mathcal{F}_{\lambda \rho}\right] \in \mathbb{Z} .
$$

Here BZ stands for the 4D Brillouin zone (a 4D torus), $\epsilon^{\mu \nu \lambda \rho}$ is the antisymmetric tensor of rank 4 , and $\mathcal{F}_{\mu \nu}$ is a matrix defined by

$$
\begin{aligned}
\mathcal{F}_{\mu \nu}^{\alpha \beta} & =\partial_{\mu} \mathcal{A}_{\nu}^{\alpha \beta}-\partial_{\nu} \mathcal{A}_{\mu}^{\alpha \beta}+i\left[\mathcal{A}_{\mu}, \mathcal{A}_{\nu}\right]^{\alpha \beta}, \\
\mathcal{A}_{\mu}^{\alpha \beta}(\mathbf{k}) & =-i\left\langle\alpha, \mathbf{k}\left|\partial_{\mu}\right| \beta, \mathbf{k}\right\rangle,
\end{aligned}
$$

where $\partial_{\mu}=\partial / \partial k_{\mu},|\alpha, \mathbf{k}\rangle$ are the eigenstates of the $\alpha$ th band, and the indices $\alpha$ and $\beta$ run over all the bands below the gap. It is alternatively expressed as [21]

$$
C_{i j}^{(2)}=-\frac{1}{8 \pi^{2}} \int_{\mathrm{BZ}} d^{4} k \epsilon^{\mu \nu \lambda \rho} \operatorname{Tr}\left[P \frac{\partial P}{\partial k_{\mu}} \frac{\partial P}{\partial k_{\nu}} P \frac{\partial P}{\partial k_{\lambda}} \frac{\partial P}{\partial k_{\rho}}\right],
$$

where $P(\mathbf{k})=\sum_{\alpha \in \text { occ }}|\alpha, \mathbf{k}\rangle\langle\alpha, \mathbf{k}|$ is the projection operator to the eigenstates below the gap. Note that we have six second Chern numbers depending on the choice of $i, j(i \neq j)$ from $1,2,3,4$. 
When the Fermi energy is in the gap, the electromagnetic response of the $4 \mathrm{D}$ system is given by [21,62-67]

$$
j_{\mu}^{(4 \mathrm{D})}=\frac{e^{3}}{h^{2}} C_{i j}^{(2)} \epsilon^{\mu \nu \lambda \rho} B_{v \lambda} E_{\rho},
$$

where $j_{\mu}^{(4 \mathrm{D})}$ is the electric current density in $4 \mathrm{D}$ space. If a weak electric field $E_{i}$ is applied to the system, the wave number $k_{i}$ is adiabatically changed to $k_{i}+(e / \hbar) A_{i}(t)$, where $E_{i}=-\partial A_{i} / \partial t$. When we consider a cyclic process where $\phi_{i}=k_{i} a_{i}$ is changed from 0 to $2 \pi$ in a time period $T$, the corresponding electric field should be

$$
E_{i}=-\frac{h}{e a_{i}} \frac{1}{T} .
$$

According to Eq. (38), $E_{i}$ induces an electric current $\left(j_{x}, j_{y}\right)^{(4 \mathrm{D})}=\left(e^{3} / h^{2}\right) C_{i j}^{(2)}\left(-B_{y j}, B_{x j}\right) E_{i}$. The corresponding $2 \mathrm{D}$ current density per single layer is given by $j_{\mu}^{(2 \mathrm{D})}=$ $j_{\mu}^{(4 \mathrm{D})} a_{i} a_{j}$, giving

$$
\left(j_{x}, j_{y}\right)^{(2 \mathrm{D})}=-\frac{e}{2 \pi} C_{i j}^{(2)}\left(b_{j, y},-b_{j, x}\right) \frac{1}{T},
$$

where we used Eqs. (34) and (39). Total polarization change in the process $\left(\phi_{i}: 0 \rightarrow 2 \pi\right)$ is $\Delta \mathbf{P}_{i}=\mathbf{j}^{(2 \mathrm{D})} T /(-e)$. By appending the summation over $j$, we finally obtain

$$
\Delta \mathbf{P}_{i}=-\frac{1}{2 \pi} \sum_{j} C_{i j}^{(2)}\left(\mathbf{b}_{j} \times \mathbf{e}_{z}\right) .
$$

We notice that Eq. (41) has the exact same form as Eq. (25). By comparing the two equations, we immediately find

$$
v_{i j}=-C_{i j}^{(2)},
$$

i.e., the zone quantum numbers turned out to be the second Chern numbers.

By using Eq. (37), we numerically calculated $C_{i j}^{(2)}$ for some of the commensurate approximants in the twisted triangular potential series considered in Sec. II B, and we confirmed the agreement with $-v_{i j}$. Since Eq. (37) includes the integral on the Brillouin zone of the commensurate approximant, one might think that $C_{i j}^{(2)}$ depends explicitly on the Brillouin zone size (inverse of the commensurate unit cell size), which is rather arbitrary as seen in Appendix A. But in reality, the integrand $\operatorname{Tr}[\cdots]$ itself is proportional to the number of subbands below the gap (proportional to the unit cell size), and this cancels with the Brillouin zone integral, giving the invariant integers independent of the commensurate period.

\section{CONCLUSION}

We have shown that energy gaps in two-dimensional double-periodic systems can be uniquely labeled by six second Chern numbers. Physically, these numbers can be interpreted as zone quantum numbers, which quantize the momentum space in units of the six fundamental Brillouin zones defined in the redundant periodicities. At the same time, the zone quantum numbers also describe the quantized charge pumping under a relative slide of different periodic potentials. By considering a mapping of the 2D charge pumping to the fictitious 4D quantum Hall effect, we found that the zone quantum numbers are equivalent to the second Chern numbers.

The topological characterization of energy gaps presented in this work is applicable to any quasiperiodic systems having redundant reciprocal vectors more than the spatial dimension. If we have $n$ independent reciprocal-lattice vectors in a $d$-dimensional space, we have ${ }_{n} C_{d}$ different choices of the fundamental Brillouin zones. In a twisted multilayer system composed of $n$ layers, for instance, $2 n$ reciprocal vectors define $n(2 n-1)$ independent Brillouin zones, so that there should be $n(2 n-1)$ zone quantum numbers. The Penrose tile $[68,69]$ has five reciprocal-lattice vectors, giving the 10 quantum numbers. The extension to 3D quasicrystal should also be possible. Lastly, nonzero quantum numbers for adiabatic pumping generally implies the existence of the edge localized states $[11,21,70,71]$. The study of the edge states in general quasiperiodic systems in terms of the zone quantum numbers would also be intriguing.

\section{ACKNOWLEDGMENTS}

This work was supported in part by JSPS KAKENHI Grants No. JP20H01840, No. JP20H00127, No. JP21H05236, No. JP21H05232, and by JST CREST Grant No. JPMJCR20T3, Japan.

\section{APPENDIX A: COMMENSURATE APPROXIMANT METHOD}

We describe the commensurate approximant method to calculate the band structures and the zone quantum numbers in the double-period system. In an incommensurate case, we always have lattice points of the two periodic potentials, which happen to be very close to each other. The situation is expressed as

$$
p_{1} \mathbf{a}_{1}^{\alpha}+p_{2} \mathbf{a}_{2}^{\alpha}=p_{3} \mathbf{a}_{1}^{\beta}+p_{4} \mathbf{a}_{2}^{\beta}+\Delta \mathbf{L},
$$

where $p_{i}(i=1,2,3,4)$ are integers and $\Delta \mathbf{L}$ is the difference.

A commensurate approximant can be obtained by choosing two such nearly commensurate points [with integers $\left(p_{1}, p_{2}, p_{3}, p_{4}\right)$ and $\left.\left(q_{1}, q_{2}, q_{3}, q_{4}\right)\right]$ and deforming the potential $\beta$ such that $\Delta \mathbf{L}$ becomes zero. The two points then become the exact primitive lattice vectors of the commensurate approximant,

$$
\begin{aligned}
\left(\begin{array}{l}
\mathbf{a}_{1}^{\mathrm{c}} \\
\mathbf{a}_{2}^{\mathrm{c}}
\end{array}\right) & =\left(\begin{array}{ll}
p_{1} & p_{2} \\
q_{1} & q_{2}
\end{array}\right)\left(\begin{array}{l}
\mathbf{a}_{1}^{\alpha} \\
\mathbf{a}_{2}^{\alpha}
\end{array}\right) \\
& =\left(\begin{array}{ll}
p_{3} & p_{4} \\
q_{3} & q_{4}
\end{array}\right)\left(\begin{array}{l}
\mathbf{a}_{1}^{\beta} \\
\mathbf{a}_{2}^{\beta}
\end{array}\right) .
\end{aligned}
$$

Correspondingly, the reciprocal superlattice vectors $\mathbf{b}_{1}^{\mathrm{c}}, \mathbf{b}_{2}^{\mathrm{c}}$ are given by

$$
\begin{aligned}
\left(\begin{array}{l}
\mathbf{b}_{1}^{\mathrm{c}} \\
\mathbf{b}_{2}^{\mathrm{c}}
\end{array}\right) & =\left[\left(\begin{array}{ll}
p_{1} & p_{2} \\
q_{1} & q_{2}
\end{array}\right)^{T}\right]^{-1}\left(\begin{array}{l}
\mathbf{b}_{1}^{\alpha} \\
\mathbf{b}_{2}^{\alpha}
\end{array}\right) \\
& =\left[\left(\begin{array}{ll}
p_{3} & p_{4} \\
q_{3} & q_{4}
\end{array}\right)^{T}\right]^{-1}\left(\begin{array}{l}
\mathbf{b}_{1}^{\beta} \\
\mathbf{b}_{2}^{\beta}
\end{array}\right),
\end{aligned}
$$


(a) $29.4093^{\circ}$

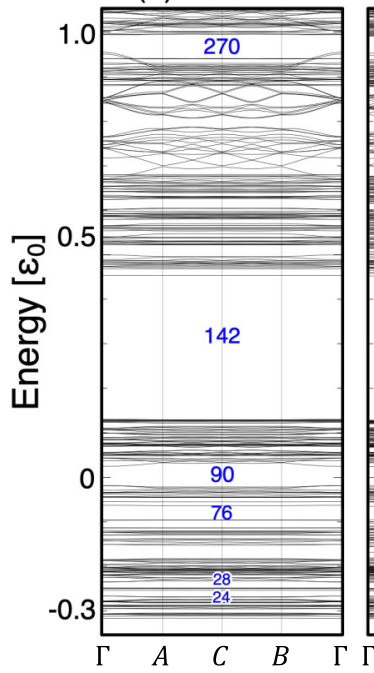

(b) $29.5046^{\circ}$

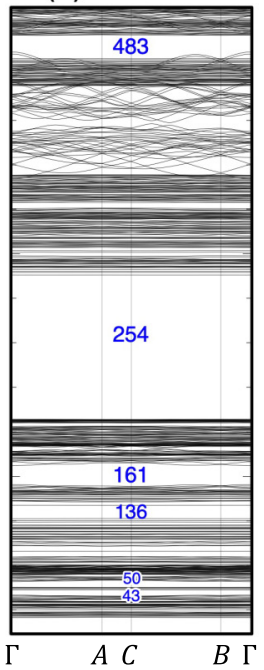

(c) $29.6566^{\circ}$

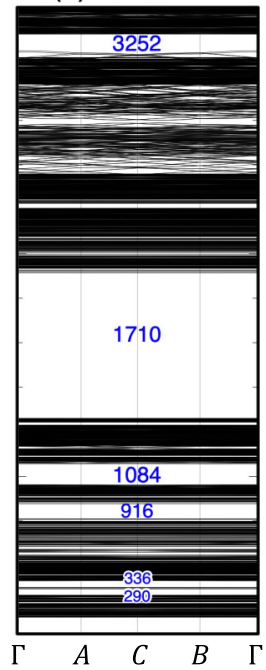

(d) $29.8417^{\circ}$

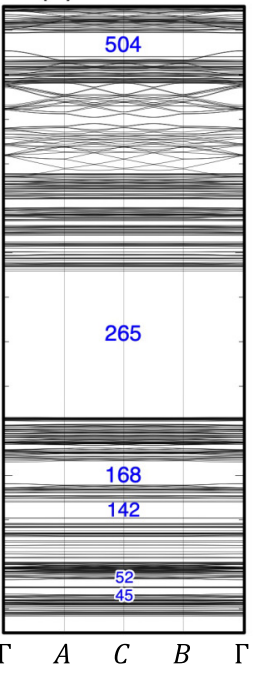

(e) $29.9576^{\circ}$

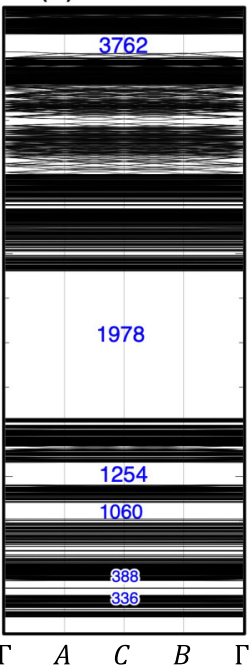

(f) $30.0579^{\circ}$

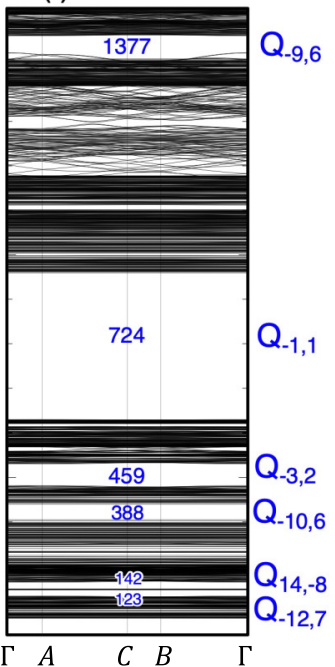

FIG. 7. Band structures of commensurate approximants (a)-(f) (specified in Table I) for the twisted double triangular potential near $30^{\circ}$. The Brillouin zone path is taken as $(\Gamma, A, C, B, \Gamma) \equiv\left(0, \mathbf{b}_{1}^{\mathrm{c}} / 2,\left(\mathbf{b}_{1}^{\mathrm{c}}+\mathbf{b}_{2}^{\mathrm{c}}\right) / 2, \mathbf{b}_{2}^{\mathrm{c}} / 2,0\right)$. The integers in gaps indicate the number of bands below the gap, $r$.

where $T$ stands for the matrix transpose. $\mathbf{a}_{\mu}^{\mathrm{c}}$ and $\mathbf{b}_{\mu}^{\mathrm{c}}$ are related by

$$
\mathbf{a}_{1}^{\mathrm{c}}=\frac{S_{\mathrm{c}}}{2 \pi}\left(\mathbf{b}_{2}^{\mathrm{c}} \times \mathbf{e}_{z}\right), \quad \mathbf{a}_{2}^{\mathrm{c}}=-\frac{S_{\mathrm{c}}}{2 \pi}\left(\mathbf{b}_{1}^{\mathrm{c}} \times \mathbf{e}_{z}\right),
$$

where $S_{\mathrm{c}}=\left(\mathbf{a}_{1}^{\mathrm{c}} \times \mathbf{a}_{2}^{\mathrm{c}}\right)_{z}$ is the unit area of the commensurate approximant.

By using the serial notation Eq. (3), Equation (A3) can simply be written as

$$
\mathbf{b}_{i}=p_{i} \mathbf{b}_{1}^{\mathrm{c}}+q_{i} \mathbf{b}_{2}^{\mathrm{c}} .
$$

Accordingly, the unit areas Eq. (5) become

$$
S_{i j}^{*}=\left(p_{i} q_{j}-p_{j} q_{i}\right) S_{\mathrm{c}}^{*},
$$

where $S_{\mathrm{c}}^{*}=\left(\mathbf{b}_{1}^{\mathrm{c}} \times \mathbf{b}_{2}^{\mathrm{c}}\right)_{z}=(2 \pi)^{2} / S_{\mathrm{c}}$ is the area of the first Brillouin zone of the commensurate approximant. Equation (4) becomes the Diophantine equation,

$$
r=\sum_{\langle i, j\rangle} v_{i j}\left(p_{i} q_{j}-p_{j} q_{i}\right)
$$

where $r \equiv n_{e} /\left[S_{\mathrm{c}}^{*} /(2 \pi)^{2}\right]$ is for the number of bands below the gap.

In determination of the zone quantum numbers $v_{i j}$, we consider a series of commensurate approximants near the target system, and we solve a set of Diophantine equations (A7) for all the approximants. As an example, we show in Fig. 7 the band structures of six commensurate approximants (a)-(f) for the double triangular potential near $\theta=30^{\circ}$ (see Fig. 3), which are specified by $\left(p_{1}, p_{2}, p_{3}, p_{4} ; q_{1}, q_{2}, q_{3}, q_{4}\right)$ in Table I. The Brillouin zone path is taken as $(\Gamma, A, C, B, \Gamma) \equiv$ $\left(0, \mathbf{b}_{1}^{\mathrm{c}} / 2,\left(\mathbf{b}_{1}^{\mathrm{c}}+\mathbf{b}_{2}^{\mathrm{c}}\right) / 2, \mathbf{b}_{2}^{\mathrm{c}} / 2,0\right)$. Table I also shows the number of occupied bands $r$ for some major gaps $Q_{m, n}$. The six systems have very close potential profiles and similar spectral structures, while they have completely different sizes of the commensurate unit cells and thus different numbers of bands below the same gap. For the largest gap $Q_{-1,1}$, for instance, the numbers of the bands are $r=142,254,1710,265,1978,724$ for the six systems, and accordingly we have six independent equations of Eq. (A7) with six unknown variables $v_{i j}$. By solving the set of equations, we find $v_{i j}=(-1,1,2,-1,1,-1)$ as a unique solution. All other approximants sharing the same gap have the same solution of $v_{i j}$.

The formula of quantum pumping Eq. (25) can also be transformed to the commensurate version. By using Eq. (A5), Eq. (25) is written as

$$
\Delta \mathbf{P}_{i}=\frac{1}{2 \pi} \sum_{j(\neq i)} v_{i j}\left[p_{j}\left(\mathbf{b}_{1}^{\mathrm{c}} \times \mathbf{e}_{z}\right)+q_{j}\left(\mathbf{b}_{2}^{\mathrm{c}} \times \mathbf{e}_{z}\right)\right] .
$$

\begin{tabular}{|c|c|c|c|c|c|c|c|c|c|c|c|c|c|c|c|}
\hline & $\theta$ & $p_{1}$ & $p_{2}$ & $p_{3}$ & $p_{4}$ & $q_{1}$ & $q_{2}$ & $q_{3}$ & $q_{4}$ & $r\left[Q_{-12,7}\right]$ & $r\left[Q_{-14,-8}\right]$ & $r\left[Q_{-10,6}\right]$ & $r\left[Q_{-3,2}\right]$ & $r\left[Q_{-1,1}\right]$ & $r\left[Q_{-9,6}\right]$ \\
\hline (a) & 29.4093 & 3 & 8 & 8 & 3 & -8 & 11 & -3 & 11 & 24 & 28 & 76 & 90 & 142 & 270 \\
\hline (b) & 29.5046 & 3 & 8 & 8 & 3 & -9 & 34 & 9 & 25 & 43 & 50 & 136 & 161 & 254 & 483 \\
\hline (c) & 29.6566 & 25 & 9 & 34 & -9 & -27 & 37 & -10 & 37 & 290 & 336 & 916 & 1084 & 1710 & 3252 \\
\hline (d) & 29.8417 & 11 & 4 & 15 & -4 & -4 & 15 & 4 & 11 & 45 & 52 & 142 & 168 & 265 & 504 \\
\hline (e) & 29.9576 & 11 & 30 & 30 & 11 & -30 & 41 & -11 & 41 & 336 & 388 & 1060 & 1254 & 1978 & 3762 \\
\hline (f) & 30.0579 & 11 & 30 & 30 & 11 & -11 & 15 & -4 & 15 & 123 & 142 & 388 & 459 & 724 & 1377 \\
\hline
\end{tabular}

TABLE I. Twist angle $\theta$ and the indices $\left(p_{1}, p_{2}, p_{3}, p_{4} ; q_{1}, q_{2}, q_{3}, q_{4}\right)$ of the commensurate approximants (a)-(f). The $r\left[Q_{m, n}\right]$ is the number of the occupied bands below the gap $Q_{m, n}$. 
(a) Single potential

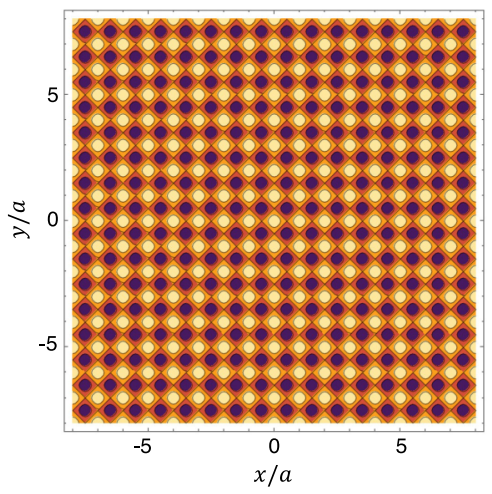

(b) $\theta=7^{\circ}$

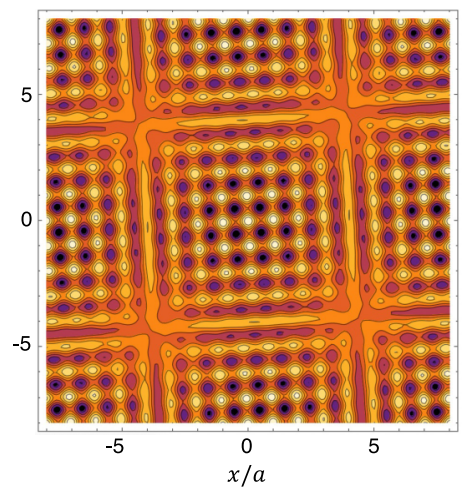

(c) $\theta=45^{\circ}$

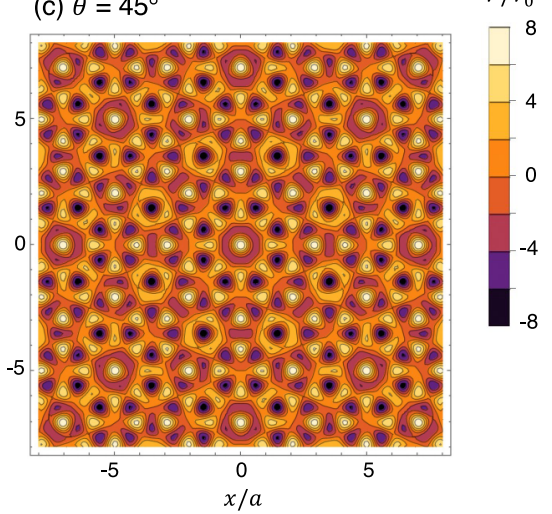

FIG. 8. Contour plots of (a) single square potential, and (b) twisted double square potential with $\theta=7^{\circ}$ and (c) $\theta=45^{\circ}$ [Eq. (B1)].

By using Eq. (A4), it is reduced to

$$
\Delta \mathbf{P}_{i}=\frac{1}{S_{\mathrm{c}}}\left(C_{i 1} \mathbf{a}_{1}^{\mathrm{c}}+C_{i 2} \mathbf{a}_{2}^{\mathrm{c}}\right)
$$

where

$$
C_{i 1}=\sum_{j(\neq i)} v_{i j} q_{j}, \quad C_{i 2}=-\sum_{j(\neq i)} v_{i j} p_{j}
$$

are the integers to characterize the pumping in units of the commensurate period. By using Eqs. (A10) and (A7), we have the Diophantine equation for $C_{i l}$ 's,

$$
\begin{array}{ll}
\sum_{i=1}^{4} p_{i} C_{i 1}=r, & \sum_{i=1}^{4} p_{i} C_{i 2}=0, \\
\sum_{i=1}^{4} q_{i} C_{i 1}=0, & \sum_{i=1}^{4} q_{i} C_{i 2}=-r,
\end{array}
$$

which agrees with the results in the previous work [26].

The integers $C_{i 1}$ and $C_{i 2}$ are expressed as the first Chern numbers [26-28]. The Bloch Hamiltonian for the commensurate approximant can be written as $H\left(k_{1}, k_{2} ; \phi_{1}, \phi_{2}, \phi_{3}, \phi_{4}\right)$, where $k_{l}=\mathbf{k} \cdot \mathbf{a}_{l}^{c} /\left|\mathbf{a}_{l}^{c}\right|(l=1,2)$ is the component of the Bloch wave vector along $\mathbf{a}_{l}^{c}$, and $\phi_{i}(i=1,2,3,4)$ is the phase factors for the potential slide [Eq. (21)]. Then $C_{i l}$ is given by the first Chern number on a $2 \mathrm{D}$ torus of $\left(k_{l}, \phi_{i}\right)$, or

$$
C_{i l}=\frac{1}{2 \pi} \int_{0}^{\left|\mathbf{b}_{l}^{c}\right|} d k_{l} \int_{0}^{2 \pi} d \phi_{i} F_{i l} \in \mathbb{Z},
$$

where $F_{i l}$ is the Berry curvature defined by

$$
\begin{aligned}
F_{i l} & =\partial_{1} a^{(2)}-\partial_{2} a^{(1)}, \\
a^{(\mu)} & =-i \sum_{n \in \mathrm{occ}}\left\langle\alpha, \mathbf{k}\left|\partial_{\mu}\right| \alpha, \mathbf{k}\right\rangle,
\end{aligned}
$$

and $\partial_{1}=\partial / \partial k_{l}$ and $\partial_{2}=\partial / \partial \phi_{i}$. The integral period of $k(0 \leqslant$ $k \leqslant\left|\mathbf{b}_{l}^{c}\right|$ ) in Eq. (A12) represents the span of the first Brillouin zone in the $l$ direction.

Unlike the second Chern number $v_{i j}$, the first Chern number $C_{i l}$ of the 2D commensurate system depends directly on the unit-cell size and it is not an invariant in a continuous deformation. In Eq. (A10), indeed, $C_{i j}$ depends on $p_{i}$ and $q_{i}$, and hence the systems in Fig. 7 have all different $C_{i j}$ 's for the same gap. The direct dependence of $C_{i j}$ on the unit-cell size can be understood by considering the same system with a redundant unit cell spanned by $M_{1} \mathbf{a}_{1}^{\mathrm{c}}$ and $M_{2} \mathbf{a}_{2}^{\mathrm{c}}$ with arbitrary integers $M_{1}$ and $M_{2}$. Due to the band folding, the integral path in Eq. (A12) is reduced to $0 \leqslant k_{l} \leqslant\left|\mathbf{b}_{l}^{c}\right| / M_{l}$, and the integrand $F_{i l}$ (proportional to the number of bands) is multiplied by $M_{1} M_{2}$. As a consequence, the first Chern number for the enlarged unit cell becomes $C_{i 1}^{\prime}=M_{2} C_{i 1}$ and $C_{i 2}^{\prime}=M_{1} C_{i 2}$. This is natural because the integer $C_{i 1}\left(C_{i 2}\right)$ corresponds to the number of electrons passing through the unit-cell side along $\mathbf{a}_{2}^{\mathrm{c}}\left(\mathbf{a}_{1}^{\mathrm{c}}\right)$ during a cyclic process, and hence it is just proportional to the span of the corresponding unit-cell side. In contrast, the second Chern number $v_{i j}$ [Eq. (35)] includes an integral on the whole 2D Brillouin zone ( $k_{x} k_{y}$-plane), and this cancels with the factor $M_{1} M_{2}$ in the integrand, giving an invariant independent of the unit-cell choice. Physically, $v_{i j}$ corresponds to the number of electrons passing through the side of the parallelogram spanned by $\mathbf{a}_{1}^{i j}$ and $\mathbf{a}_{2}^{i j}$ as argued in Sec. III B, which does not depend on the commensurability of the lattice periods.

\section{APPENDIX B: TWISTED SQUARE POTENTIALS}

We calculate the energy spectrum and the zone quantum numbers for a twisted double square potential. The Hamiltonian is given by Eq. (1) with

$$
V^{\lambda}(\mathbf{r})=2 V_{0} \sum_{\mu=1}^{2} \cos \left[\mathbf{b}_{\mu}^{\lambda} \cdot\left(\mathbf{r}-\mathbf{r}_{0}^{\lambda}\right)\right],
$$

where

$$
\mathbf{b}_{1}^{\alpha}=\frac{2 \pi}{a}\left(\begin{array}{l}
1 \\
0
\end{array}\right), \quad \mathbf{b}_{2}^{\alpha}=\frac{2 \pi}{a}\left(\begin{array}{l}
0 \\
1
\end{array}\right), \quad \mathbf{b}_{\mu}^{\beta}=R(\theta) \mathbf{b}_{\mu}^{\alpha} .
$$

The corresponding primitive lattice vectors are

$$
\begin{aligned}
& \mathbf{a}_{1}^{\alpha}=a\left(\begin{array}{l}
1 \\
0
\end{array}\right), \quad \mathbf{a}_{2}^{\alpha}=a\left(\begin{array}{l}
0 \\
1
\end{array}\right), \\
& \mathbf{a}_{\mu}^{\beta}=R(\theta) \mathbf{a}_{\mu}^{\alpha} .
\end{aligned}
$$

The potential profile is presented in Fig. 8 for (a) a single potential, and (b) a double potential with $\theta=7^{\circ}$ and (c) $\theta=45^{\circ}$. The system (c) is a quasicrystal with eightfold 

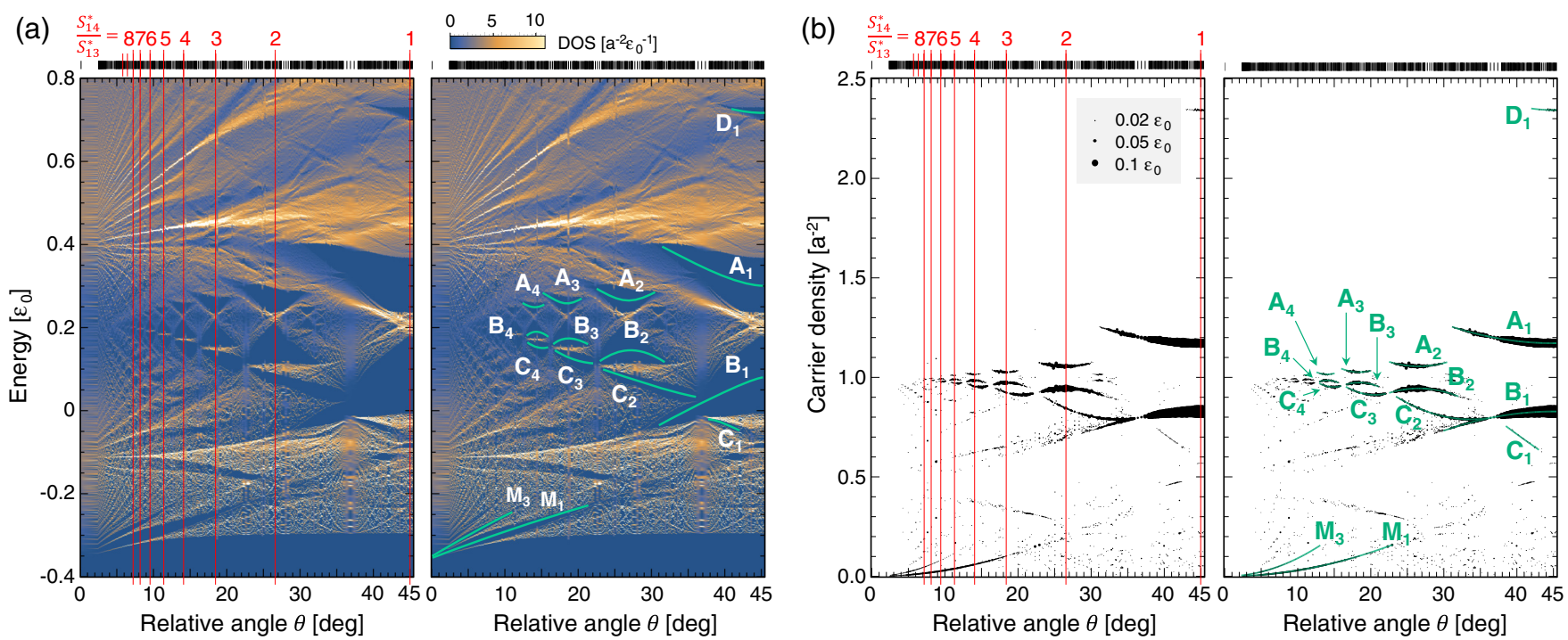

$$
\begin{array}{lllll}
M_{1}:(1,0,-1,1,0,1) & A_{4}:(17,-4,-16,16,-4,17) & A_{3}:(10,-3,-9,9,-3,10) & A_{2}:(5,-2,-4,4,-2,5) & A_{1}:(2,-1,-1,1,-1,2) \\
M_{3}:(3,0,-3,3,0,3) & B_{4}:(-16,4,16,-16,4,-16) & B_{3}:(-9,3,9,-9,3,-9) & B_{2}:(-4,2,4,-4,2,-4) & B_{1}:(-1,1,1,-1,1,-1) \\
& C_{4}:(16,-4,-15,15,-4,16) & C_{3}:(9,-3,-8,8,-3,9) & C_{2}:(4,-2,-3,3,-2,4) & C_{1}:(1,-1,0,0,-1,1) \\
& & & & \\
& & &
\end{array}
$$

FIG. 9. Similar plots to Fig. 3 calculated for the twisted double square potential.

rotational symmetry. The potential amplitude is taken as $V_{0}=$ $0.16 \varepsilon_{0}$, where $\varepsilon_{0}=\hbar^{2} /\left(2 m a^{2}\right)$.

Figure 9 is a set of plots similar to Fig. 3, calculated for the twisted square potential. The zone quantum numbers are presented in the bottom of the figure. In this case, we clearly see a series recursive gaps labeled by $A_{n}, B_{n}, C_{n}$, where

$$
\begin{aligned}
& A_{n}=(1,0,0,0,0,1)-B_{n}, \\
& B_{n}=\left(-n^{2}, n, n^{2},-n^{2}, n,-n^{2}\right), \\
& C_{n}=(0,0,1,-1,0,0,)-B_{n} .
\end{aligned}
$$

We observe that the extrema of $A_{n}$ and $B_{n}$ in the electron density coincide with the twist angle satisfying $S_{14}^{*} / S_{13}^{*}=n$. Since $S_{14}^{*} / S_{13}^{*}$ is equal to $1 / \tan \theta$ in this model, the corresponding angles are given by $\theta_{n}=\arctan (1 / n)$. Considering $S_{i j}^{*}=b^{2}(1, \sin \theta, \cos \theta,-\cos \theta, \sin \theta, 1), \theta_{n}$ corresponds to the special angles where $v_{13} S_{13}^{*}=v_{14} S_{14}^{*}=v_{23} S_{23}^{*}=v_{24} S_{24}^{*}$ is satisfied for $A_{n}$ and $B_{n}$, i.e., four out of six quantized areas happen to coincide.

We show the qBZs of the gap $A_{1}$ and $B_{1}$ at $\theta=45^{\circ}$ in Fig. 10(a). The decomposition into the primitive Brillouin zone is illustrated in the right two panels. The area of $B_{1}$ is given by $S^{*}\left(B_{1}\right)=\left(p_{1}+p_{2}\right)-q=S_{13}^{*}+S_{24}^{*}-\left(S_{23}^{*}+\right.$ $\left.S_{12}^{*}+S_{34}^{*}-S_{14}^{*}\right)$, which correctly gives the zone quantum numbers $(-1,1,1,-1,1,-1)$. The area of $A_{1}$ is $S^{*}\left(A_{1}\right)=$ $\left(g_{1}+g_{2}\right)-S^{*}\left(B_{1}\right)$, giving $(2,-1,-1,1,-1,2)$.

\section{APPENDIX C: CHARGE PUMPING FORMULA FROM THE INFINITESIMAL POTENTIAL LIMIT}

Here we present an alternative method to derive the relation of the charge pumping to the electron density, Eq. (22), by integrating the Berry curvature in the infinitesimal potential limit. First, let us consider a $1 \mathrm{D}$ system with a single periodic potential,

$$
H(\phi)=\frac{p^{2}}{2 m}+V\left(x-\frac{\phi}{2 \pi} a\right),
$$

where $V(x)$ is a periodic potential with the period of $a=$ $2 \pi / b$, and the phase $\phi$ describes sliding of the potential. If we write the periodic potential in a Fourier series as $V(x)=$ $\sum_{m} V_{m} e^{i m b x}$, the translated potential is expressed as

$$
V\left(x-\frac{\phi}{2 \pi} a\right)=\sum_{m} V_{m} e^{-i m \phi} e^{i m b x} .
$$

The electric polarization can be calculate by

$$
P(\phi)=\sum_{n \in \text { occ. }} \int_{-b / 2}^{b / 2} \frac{d k}{2 \pi} i\left\langle u_{n k}(\phi)\left|\frac{\partial}{\partial k}\right| u_{n k}(\phi)\right\rangle,
$$

where $u_{n k}(\phi)$ is the Bloch eigenstate of the $n$th band in the Hamiltonian at phase shift $\phi$, and "occ." represents the occupied bands below the Fermi energy. The charge transport during a single sliding process is given by $\Delta P=$ $\int_{0}^{2 \pi} d \phi(\partial P / \partial \phi)$. It is expressed as the Chern number on $(k, \phi)$ space,

$$
\Delta P=\sum_{n \in \mathrm{occ} .} \int_{0}^{2 \pi} d \phi \int_{-b / 2}^{b / 2} \frac{d k}{2 \pi} F_{n}(k, \phi),
$$

where $F_{n}(k, \phi)$ is the Berry curvature defined by

$$
\begin{aligned}
F_{n}(k, \phi) & =\partial_{1} a_{n}^{(2)}-\partial_{2} a_{n}^{(1)}, \\
a_{n}^{(i)}(k, \phi) & =-i\left\langle u_{n k}(\phi)\left|\partial_{i}\right| u_{n k}(\phi)\right\rangle,
\end{aligned}
$$

and $\partial_{1}=\partial / \partial k$ and $\partial_{2}=\partial / \partial \phi$. 

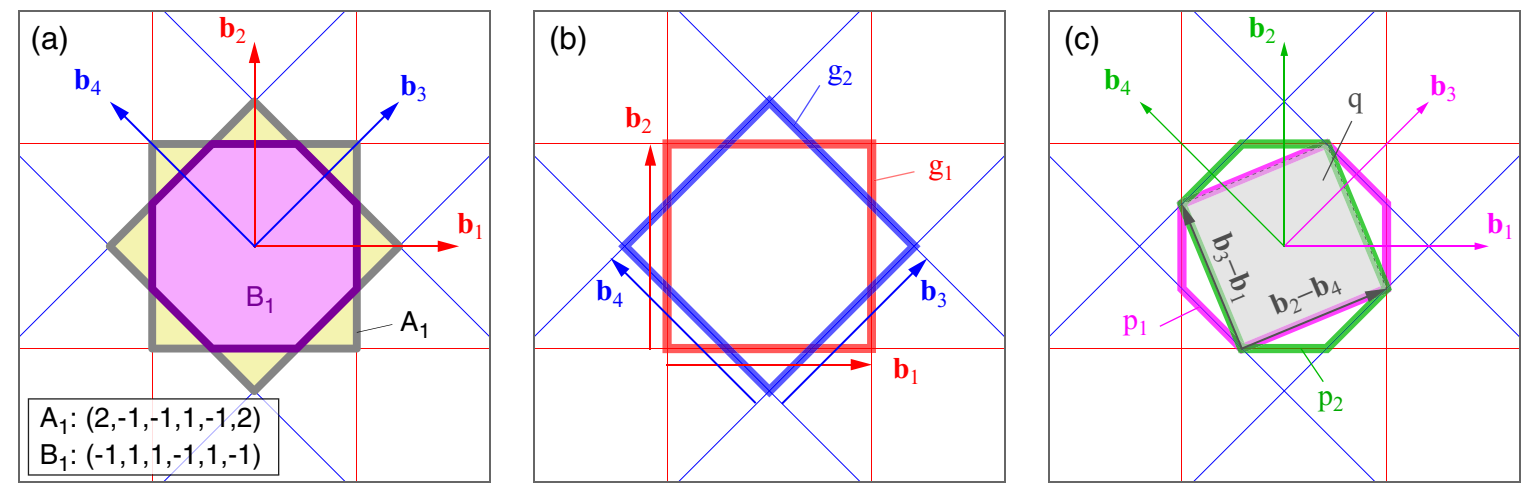

FIG. 10. (a) The qBZs of $A_{1}$ and $B_{1}$ in the twisted double square potential with $\theta=45^{\circ}$. (b),(c) Decomposition of the qBZ into the primitive Brillouin zones.

The $\Delta P$ can be easily calculated by considering an infinitesimal potential limit $V(x) \rightarrow 0$. When $V$ is slightly increased from zero, energy gaps open at $k= \pm m b / 2(m=$ $1,2,3, \ldots)$ in the original parabolic band of a free electron as illustrated in Fig. 11. Let us consider the eigenstates of the first band, $u_{1, k}$ in the first Brillouin zone $-b / 2 \leqslant k \leqslant b / 2$. It is written as $\left|u_{1, k}\right\rangle=\sum_{m} c_{m} e^{i(k+m b) x}$, and we fix the global phase such that $c_{0}$ is real. At the edges of the Brillouin zone, we have

$$
\begin{aligned}
\left|u_{1, b / 2}(\phi)\right\rangle & \approx \frac{1}{\sqrt{2}} e^{i(b / 2) x}+\frac{e^{i \phi}}{\sqrt{2}} e^{i(-b / 2) x}, \\
\left|u_{1,-b / 2}(\phi)\right\rangle & \approx \frac{e^{-i \phi}}{\sqrt{2}} e^{i(b / 2) x}+\frac{1}{\sqrt{2}} e^{i(-b / 2) x} .
\end{aligned}
$$

They are the same states but differ in the global phase factor by $e^{i \phi}$. By applying the Stokes theorem to Eq. (C4), the Chern number of the first band is just given by

$$
\Delta P=\frac{1}{2 \pi} \int_{0}^{2 \pi} d \phi\left[a_{1}^{(2)}(b / 2, \phi)-a_{1}^{(2)}(-b / 2, \phi)\right] .
$$

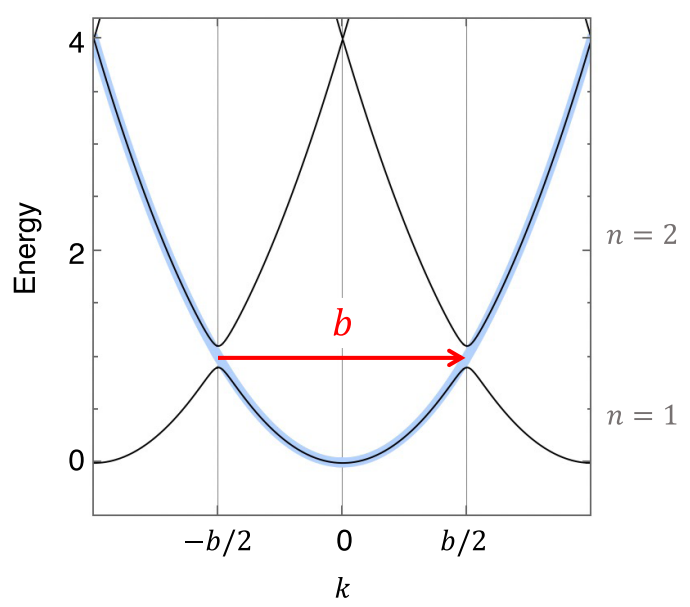

FIG. 11. Schematics of the energy band of a 1D periodic system in an infinitesimal potential limit. When the periodic potential $V$ is slightly increased from zero, energy gaps open at $k= \pm m b / 2$ ( $m=$ $1,2,3, \ldots$ ) in the original parabolic band of a free electron (blue thin curve).
Since $\left|u_{1, b / 2}(\phi)\right\rangle=e^{i \phi}\left|u_{1,-b / 2}(\phi)\right\rangle$, we find $a_{1}^{(2)}(b / 2, \phi)=$ $a_{1}^{(2)}(-b / 2, \phi)+2 \pi$, and $\Delta P=1$ is concluded. In increasing the potential $V$, the Chern numbers do not change as long as the gap remains opening.

The charge pumping for the $m$ th gap at $k= \pm m b / 2$ can also be calculated in the same manner. We note that any perturbational processes to open the $m$ th gap share the same $\phi$-dependent phase factor $e^{-i m \phi}$. For instance, the first-order process of $m$ th harmonics has an amplitude of $V_{m} e^{-i m \phi}$, and the $m$ th order process of the first harmonics is proportional to $\left(V_{1} e^{-i \phi}\right)^{m}$. We can integrate the Berry curvature for the $2 \mathrm{D}$ torus of $-m b / 2 \leqslant k \leqslant m b / 2$ and $0 \leqslant \phi \leqslant 2 \pi$ just as for the first gap. Noting that the phase factor $e^{-i \phi}$ for the first gap is just replaced with $e^{-i m \phi}$, we conclude $\Delta P=m$. Here we neglected all the gaps in the occupied states below the $m$ th gap because they do not affect the sum of the Berry curvature.

The argument also applies to a 1D doubly periodic system of Eq. (15). When the potential is increased from zero, energy gaps open at $k= \pm\left(m_{1} b_{1}+m_{2} b_{2}\right) / 2$ of the original parabolic band. The corresponding matrix element has the phase factor of $e^{-i\left(m_{1} \phi_{1}+m_{2} \phi_{2}\right)}$. The charge pumping under a unit slide of the potential $V_{i}$ is calculated by integrating the Berry curvature on ( $\left.k, \phi_{i}\right)$ space to obtain $\Delta P_{i}=m_{i}$. It agrees with the results in Sec. III A.

The argument can be extended in a straightforward manner to a 2D doubly periodic system, Eq. (1). We consider a potential sliding expressed by Eq. (19), and we calculate the change of the polarization $\Delta \mathbf{P}_{i}$ under the process changing $\phi_{i}(i=$ $1,2,3,4)$ from 0 to $2 \pi$. As argued in Sec. II C, each energy gap of the system is associated with a $\mathrm{qBZ}$ in the infinitesimal potential limit. When the potential is slightly increased from zero, the band anticrossing occurs at the boundary of qBZ.

We can integrate the Berry phase accumulated near the gap in a similar manner to the $1 \mathrm{D}$ case. Let us consider a general qBZ composed of the composite Bragg line segments $q_{1}, q_{2}, \ldots, q_{N} ; \bar{q}_{1}, \bar{q}_{2}, \ldots, \bar{q}_{N}$ as illustrated in Fig. 12(a), where $q_{n}$ and $\bar{q}_{n}$ are a symmetric pair connected by the reciprocal-lattice vector. For illustration, we take $Q_{-1,1}$ of the $30^{\circ}$ case just as an example. We take a certain pair of segments $\left(q_{n}, \bar{q}_{n}\right)$, and we calculate its contribution to $\Delta \mathbf{P}_{i}$. Let $q_{n}$ be a perpendicular bisector of $\mathbf{G}_{n}=m_{n 1} \mathbf{b}_{1}+m_{n 2} \mathbf{b}_{2}+$ $m_{n 3} \mathbf{b}_{3}+m_{n 4} \mathbf{b}_{4}$, and define $\mathbf{q}_{n}=\left(q_{n x}, q_{n y}\right)$ as a vector connecting the two ends of the segment $q_{n}$ as shown in Fig. 12(b). 


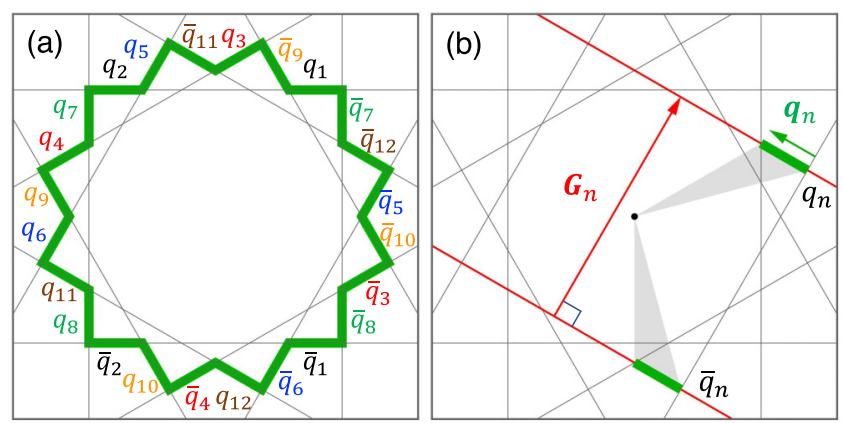

FIG. 12. (a) A qBZ boundary composed of the Bragg line segments $q_{1}, q_{2}, \ldots, q_{N} ; \bar{q}_{1}, \bar{q}_{2}, \ldots, \bar{q}_{N}$, where $q_{n}$ and $\bar{q}_{n}$ are a symmetric pair connected by the reciprocal-lattice vector $\mathbf{G}_{n}$ as shown in (b).

We choose the direction of $\mathbf{q}_{n}$ so that $\left(\mathbf{G}_{n} \times \mathbf{q}_{n}\right)_{z}>0$. The matrix element associated with the gap includes the phase factor $e^{-i\left(m_{n 1} \phi_{1}+m_{n 2} \phi_{2}+m_{n 3} \phi_{3}+m_{n 4} \phi_{4}\right)}$. Let us consider a process to change $\phi_{i}$ from 0 to $2 \pi$. Following the discussion for the $1 \mathrm{D}$ case, the corresponding polarization change along $x$ at fixed $k_{y}$ is equal to $m_{n i}$. The total polarization change contributed from the segments $\left(q_{n}, \bar{q}_{n}\right)$ is obtained by integrating it along $k_{y}$, giving $\Delta P_{i}^{(x)}=m_{n i} q_{n y} /(2 \pi)$. For the $y$ component, similarly, we have $\Delta P_{i}^{(y)}=-m_{n i} q_{n x} /(2 \pi)$. Since

[1] S. Ryu, A. P Schnyder, A. Furusaki, and A. W. W. Ludwig, Topological insulators and superconductors: tenfold way and dimensional hierarchy, New J. Phys. 12, 065010 (2010).

[2] X.-L. Qi and S.-C. Zhang, Topological insulators and superconductors, Rev. Mod. Phys. 83, 1057 (2011).

[3] D. J. Thouless, M. Kohmoto, M. P. Nightingale, and M. den Nijs, Quantized Hall Conductance in a Two-Dimensional Periodic Potential, Phys. Rev. Lett. 49, 405 (1982).

[4] M. Kohmoto, Topological invariant and the quantization of the hall conductance, Ann. Phys. 160, 343 (1985).

[5] M. E. Zoorob, M. D. B. Charlton, G. J. Parker, J. J. Baumberg, and M. C. Netti, Complete photonic bandgaps in 12-fold symmetric quasicrystals, Nature (London) 404, 740 (2000).

[6] M. A. Kaliteevski, S. Brand, R. A. Abram, T. F. Krauss, R. DeLa Rue, and P. Millar, Two-dimensional penrose-tiled photonic quasicrystals: from diffraction pattern to band structure, Nanotechnology 11, 274 (2000).

[7] P. N. Dyachenko, Y. V Miklyaev, and V. E. Dmitrienko, Threedimensional photonic quasicrystal with a complete band gap, JETP Lett. 86, 240 (2007).

[8] M. Krajčí and J. Hafner, Topologically induced semiconductivity in icosahedral al-pd-re and its approximants, Phys. Rev. B 75, 024116 (2007).

[9] L.-J. Lang, X. Cai, and S. Chen, Edge States and Topological Phases in One-Dimensional Optical Superlattices, Phys. Rev. Lett. 108, 220401 (2012).

[10] F. Mei, S.-L. Zhu, Z.-M. Zhang, C. H. Oh, and N. Goldman, Simulating $\mathrm{Z}_{2}$ topological insulators with cold atoms in a onedimensional optical lattice, Phys. Rev. A 85, 013638 (2012).

[11] Y. E. Kraus, Y. Lahini, Z. Ringel, M. Verbin, and O. Zilberberg, Topological States and Adiabatic Pumping in Quasicrystals, Phys. Rev. Lett. 109, 106402 (2012). $\mathbf{q}_{n}$ is perpendicular to $\mathbf{G}_{n}$, it is written in vector form as $\Delta \mathbf{P}_{i}=m_{n i}\left|\mathbf{q}_{n}\right|\left(\mathbf{G}_{n} /\left|\mathbf{G}_{n}\right|\right) /(2 \pi)$. By taking a summation over all the segments of the $\mathrm{qBZ}$, we obtain

$$
\Delta \mathbf{P}_{i}=\sum_{n} \frac{1}{2 \pi} m_{n i}\left|\mathbf{q}_{n}\right| \frac{\mathbf{G}_{n}}{\left|\mathbf{G}_{n}\right|} .
$$

We can relate the polarization change Eq. (C8) to the derivative of the electronic density as follows. The electronic density $n_{e}$ for below the gap is given by the area of the $\mathrm{qBZ}$ divided by $(2 \pi)^{2}$. When $\mathbf{b}_{i}$ is changed by $\delta \mathbf{b}_{i}, \mathbf{G}_{n}$ changes by $\delta \mathbf{G}_{n}=m_{n i} \delta \mathbf{b}_{i}$, and it contributes to the change of the $\mathrm{qBZ}$ area by $\left|\mathbf{q}_{n}\right| \delta\left|\mathbf{G}_{n}\right|=\left|\mathbf{q}_{n}\right| \mathbf{G}_{n} \cdot\left(m_{n i} \delta \mathbf{b}_{i}\right) /\left|\mathbf{G}_{n}\right|$. As a result, the change of the electron density becomes

$$
\begin{aligned}
\delta n_{e} & =\sum_{n} \frac{1}{(2 \pi)^{2}}\left|\mathbf{q}_{n}\right| \frac{\mathbf{G}_{n} \cdot\left(m_{n i} \delta \mathbf{b}_{i}\right)}{\left|\mathbf{G}_{n}\right|} \\
& =\frac{1}{2 \pi} \Delta \mathbf{P}_{i} \cdot \delta \mathbf{b}_{i},
\end{aligned}
$$

where we used Eq. (C8). This immediately gives

$$
\frac{\partial n_{e}}{\partial \mathbf{b}_{i}}=\frac{1}{2 \pi} \Delta \mathbf{P}_{i},
$$

which is Eq. (22).
[12] Y. E. Kraus and O. Zilberberg, Topological Equivalence Between the Fibonacci Quasicrystal and the Harper Model, Phys. Rev. Lett. 109, 116404 (2012).

[13] I. I. Satija and G. G. Naumis, Chern and Majorana modes of quasiperiodic systems, Phys. Rev. B 88, 054204 (2013).

[14] S. Ganeshan, K. Sun, and S. Das Sarma, Topological ZeroEnergy Modes in Gapless Commensurate Aubry-André-Harper Models, Phys. Rev. Lett. 110, 180403 (2013).

[15] M. Verbin, O. Zilberberg, Y. E. Kraus, Y. Lahini, and Y. Silberberg, Observation of Topological Phase Transitions in Photonic Quasicrystals, Phys. Rev. Lett. 110, 076403 (2013).

[16] M. Verbin, O. Zilberberg, Y. Lahini, Y. E. Kraus, and Y. Silberberg, Topological pumping over a photonic fibonacci quasicrystal, Phys. Rev. B 91, 064201 (2015).

[17] M. Lohse, C. Schweizer, O. Zilberberg, M. Aidelsburger, and I. Bloch, A thouless quantum pump with ultracold bosonic atoms in an optical superlattice, Nat. Phys. 12, 350 (2016).

[18] P. Marra and M. Nitta, Topologically quantized current in quasiperiodic thouless pumps, Phys. Rev. Research 2, 042035(R) (2020).

[19] O. Zilberberg, Topology in quasicrystals, Opt. Mater. Express 11, 1143 (2021).

[20] M. Yoshii, S. Kitamura, and T. Morimoto, Topological charge pumping in quasiperiodic systems characterized by the Bott index, Phys. Rev. B 104, 155126 (2021).

[21] Y. E. Kraus, Z. Ringel, and O. Zilberberg, Four-Dimensional Quantum Hall Effect in a Two-Dimensional Quasicrystal, Phys. Rev. Lett. 111, 226401 (2013).

[22] D.-T. Tran, A. Dauphin, N. Goldman, and P. Gaspard, Topological Hofstadter insulators in a two-dimensional quasicrystal, Phys. Rev. B 91, 085125 (2015). 
[23] M. A. Bandres, M. C. Rechtsman, and M. Segev, Topological Photonic Quasicrystals: Fractal Topological Spectrum and Protected Transport, Phys. Rev. X 6, 011016 (2016).

[24] J. D. Cain, A. Azizi, M. Conrad, S. M. Griffin, and A. Zettl, Layer-dependent topological phase in a two-dimensional quasicrystal and approximant, Proc. Natl. Acad. Sci. (USA) 117, 26135 (2020).

[25] M. I. N. Rosa, M. Ruzzene, and E. Prodan, Topological gaps by twisting, Commun. Phys. 4, 130 (2021).

[26] M. Fujimoto, H. Koschke, and M. Koshino, Topological charge pumping by a sliding moiré pattern, Phys. Rev. B 101, 041112(R) (2020).

[27] Y. Zhang, Y. Gao, and D. Xiao, Topological charge pumping in twisted bilayer graphene, Phys. Rev. B 101, 041410(R) (2020).

[28] Y. Su and S.-Z. Lin, Topological sliding moiré heterostructure, Phys. Rev. B 101, 041113(R) (2020).

[29] D. J. Thouless, Quantization of particle transport, Phys. Rev. B 27, 6083 (1983).

[30] Q. Niu, Quantum adiabatic particle transport, Phys. Rev. B 34, 5093 (1986).

[31] J. M. B. Lopes dos Santos, N. M. R. Peres, and A. H. Castro Neto, Graphene Bilayer with a Twist: Electronic Structure, Phys. Rev. Lett. 99, 256802 (2007).

[32] E. J. Mele, Commensuration and interlayer coherence in twisted bilayer graphene, Phys. Rev. B 81, 161405(R) (2010).

[33] G. Trambly de Laissardière, D. Mayou, and L. Magaud, Localization of dirac electrons in rotated graphene bilayers, Nano Lett. 10, 804 (2010).

[34] S. Shallcross, S. Sharma, E. Kandelaki, and O. A. Pankratov, Electronic structure of turbostratic graphene, Phys. Rev. B 81, 165105 (2010).

[35] E. S. Morell, J. D. Correa, P. Vargas, M. Pacheco, and Z. Barticevic, Flat bands in slightly twisted bilayer graphene: Tight-binding calculations, Phys. Rev. B 82, 121407(R) (2010).

[36] R. Bistritzer and A. H. MacDonald, Moiré bands in twisted double-layer graphene, Proc. Natl. Acad. Sci. (USA) 108, 12233 (2011).

[37] P. Moon and M. Koshino, Energy spectrum and quantum Hall effect in twisted bilayer graphene, Phys. Rev. B 85, 195458 (2012).

[38] G. T. de Laissardiere, D. Mayou, and L. Magaud, Numerical studies of confined states in rotated bilayers of graphene, Phys. Rev. B 86, 125413 (2012).

[39] C. R. Dean, A. F. Young, I. Meric, C. Lee, L. Wang, S. Sorgenfrei, K. Watanabe, T. Taniguchi, P. Kim, K. L. Shepard, and J. Hone, Boron nitride substrates for high-quality graphene electronics, Nat. Nanotechnol. 5, 722 (2010).

[40] L. A. Ponomarenko, R. V. Gorbachev, G. L. Yu, D. C. Elias, R. Jalil, A. A. Patel, A. Mishchenko, A. S. Mayorov, C. R. Woods, J. R. Wallbank, M. Mucha-Kruczynski, B. A. Piot, M. Potemski, I. V. Grigorieva, K. S. Novoselov, F. Guinea, V. I. Fal'ko, and A. K. Geim, Cloning of dirac fermions in graphene superlattices, Nature (London) 497, 594 (2013).

[41] B. Hunt, J. D. Sanchez-Yamagishi, A. F. Young, M. Yankowitz, B. J. LeRoy, K. Watanabe, T. Taniguchi, P. Moon, M. Koshino, P. Jarillo-Herrero, and R. C. Ashoori, Massive dirac fermions and hofstadter butterfly in a van der waals heterostructure, Science 340, 1427 (2013).

[42] Y. Cao, V. Fatemi, S. Fang, K. Watanabe, T. Taniguchi, E. Kaxiras, and P. Jarillo-Herrero, Unconventional superconduc- tivity in magic-angle graphene superlattices, Nature (London) 556, 43 (2018).

[43] Y. Cao, V. Fatemi, A. Demir, S. Fang, S. L. Tomarken, J. Y. Luo, J. D. Sanchez-Yamagishi, K. Watanabe, T. Taniguchi, E. Kaxiras, R. C. Ashoori, and P. Jarillo-Herrero, Correlated insulator behaviour at half-filling in magic-angle graphene superlattices, Nature (London) 556, 80 (2018).

[44] L. Zou, H. C. Po, A. Vishwanath, and T. Senthil, Band structure of twisted bilayer graphene: Emergent symmetries, commensurate approximants, and Wannier obstructions, Phys. Rev. B 98, 085435 (2018).

[45] M. Koshino, N. F. Q. Yuan, T. Koretsune, M. Ochi, K. Kuroki, and L. Fu, Maximally Localized Wannier Orbitals and the Extended Hubbard Model for Twisted Bilayer Graphene, Phys. Rev. X 8, 031087 (2018).

[46] L. Balents, C. R. Dean, D. K. Efetov, and A. F. Young, Superconductivity and strong correlations in moiré flat bands, Nat. Phys. 16, 725 (2020).

[47] P. Stampfli, A dodecagonal quasiperiodic lattice in two dimensions, Helv. Phys. Acta 59, 1260 (1986)

[48] S. J. Ahn, P. Moon, T.-H. Kim, H.-W. Kim, H.-C. Shin, E. H. Kim, H. W. Cha, S.-J. Kahng, P. Kim, M. Koshino et al., Dirac electrons in a dodecagonal graphene quasicrystal, Science 361, 782 (2018)

[49] W. Yao, E. Wang, C. Bao, Y. Zhang, K. Zhang, K. Bao, C. K. Chan, C. Chen, J. Avila, M. C. Asensio et al., Quasicrystalline 30 twisted bilayer graphene as an incommensurate superlattice with strong interlayer coupling, Proc. Natl. Acad. Sci. (USA) 115, 6928 (2018).

[50] P. Moon, M. Koshino, and Y.-W. Son, Quasicrystalline electronic states in $30^{\circ}$ rotated twisted bilayer graphene, Phys. Rev. B 99, 165430 (2019).

[51] J. A. Crosse and P. Moon, Quasicrystalline electronic states in twisted bilayers and the effects of interlayer and sublattice symmetries, Phys. Rev. B 103, 045408 (2021).

[52] H. Ha and B.-J. Yang, Macroscopically degenerate localized zero-energy states of quasicrystalline bilayer systems in the strong coupling limit, Phys. Rev. B 104, 165112 (2021).

[53] H. Oka and M. Koshino, Fractal energy gaps and topological invariants in hbn/graphene/hbn double moiré systems, Phys. Rev. B 104, 035306 (2021).

[54] N. R Finney, M. Yankowitz, L. Muraleetharan, K. Watanabe, T. Taniguchi, C. R. Dean, and J. Hone, Tunable crystal symmetry in graphene-boron nitride heterostructures with coexisting moiré superlattices, Nat. Nanotechnol. 14, 1029 (2019).

[55] L. Wang, S. Zihlmann, M.-H. Liu, P. Makk, K. Watanabe, T. Taniguchi, A. Baumgartner, and C. Schönenberger, New generation of moiré superlattices in doubly aligned hBN/graphene/hBN heterostructures, Nano Lett. 19, 2371 (2019).

[56] Z. Wang, Y. B. Wang, J. Yin, E. Tóvári, Y. Yang, L. Lin, M. Holwill, J. Birkbeck, D. J. Perello, S. Xu et al., Composite super-moiré lattices in double-aligned graphene heterostructures, Sci. Adv. 5, eaay8897 (2019).

[57] Y. Yang, J. Li, J. Yin, S. Xu, C. Mullan, T. Taniguchi, K. Watanabe, A. K. Geim, K. S. Novoselov, and A. Mishchenko, In situ twistronics of van der waals heterostructures, Sci. Adv. 6, eabd3655 (2020). 
[58] M. Onodera, K. Kinoshita, R. Moriya, S. Masubuchi, K. Watanabe, T. Taniguchi, and T. Machida, Cyclotron resonance study of monolayer graphene under double moiré potentials, Nano Lett. 20, 4566 (2020).

[59] M. Kuiri, S. K. Srivastav, S. Ray, K. Watanabe, T. Taniguchi, T. Das, and A. Das, Enhanced electron-phonon coupling in doubly aligned hexagonal boron nitride bilayer graphene heterostructure, Phys. Rev. B 103, 115419 (2021).

[60] M. Andelkovic, S. P. Milovanovic, L. Covaci, and F. M. Peeters, Double moiré with a twist: Supermoiré in encapsulated graphene, Nano Lett. 20, 979 (2020).

[61] N. Leconte and J. Jung, Commensurate and incommensurate double moire interference in graphene encapsulated by hexagonal boron nitride, 2D Mater. 7, 031005 (2020).

[62] X.-L. Qi, T. L. Hughes, and S.-C. Zhang, Topological field theory of time-reversal invariant insulators, Phys. Rev. B 78, 195424 (2008).

[63] H. M. Price, O. Zilberberg, T. Ozawa, I. Carusotto, and N. Goldman, Four-Dimensional Quantum Hall Effect with Ultracold Atoms, Phys. Rev. Lett. 115, 195303 (2015).

[64] H. M. Price, O. Zilberberg, T. Ozawa, I. Carusotto, and N. Goldman, Measurement of Chern numbers through center-of-mass responses, Phys. Rev. B 93, 245113 (2016).

[65] M. Lohse, C. Schweizer, H. M. Price, O. Zilberberg, and I. Bloch, Exploring 4d quantum hall physics with a $2 \mathrm{~d}$ topological charge pump, Nature (London) 553, 55 (2018).

[66] O. Zilberberg, S. Huang, J. Guglielmon, M. Wang, K. P. Chen, Y. E. Kraus, and M. C. Rechtsman, Photonic topological boundary pumping as a probe of $4 \mathrm{~d}$ quantum hall physics, Nature (London) 553, 59 (2018).

[67] L. Lu, H. Gao, and Z. Wang, Topological one-way fiber of second chern number, Nat. Commun. 9, 5384 (2018).

[68] R. Penrose, The role of aesthetics in pure and applied mathematical research, Bull. Inst. Math. Appl. 10, 266 (1974).

[69] S. Walter and S. Deloudi, Crystallography of Quasicrystals: Concepts, Methods and Structures (Springer-Verlag, Berlin, Heidelberg, 2009), Vol. 126.

[70] Y. Hatsugai and T. Fukui, Bulk-edge correspondence in topological pumping, Phys. Rev. B 94, 041102(R) (2016).

[71] M. Fujimoto and M. Koshino, Moiré edge states in twisted bilayer graphene and their topological relation to quantum pumping, Phys. Rev. B 103, 155410 (2021). 